\title{
Multi-Antenna Aided Secrecy Beamforming Optimization for Wirelessly Powered HetNets
}

\author{
Shiqi Gong, Shaodan Ma, Chengwen Xing, Yonghui Li, Fellow, IEEE, and Lajos Hanzo, Fellow, IEEE
}

\begin{abstract}
The new paradigm of wirelessly powered two-tier heterogeneous networks (HetNets) is considered in this paper. Specifically, the femtocell base station (FBS) is powered by a power beacon $(\mathrm{PB})$ and transmits confidential information to a legitimate femtocell user (FU) in the presence of a potential eavesdropper (EVE) and a macro base station (MBS). In this scenario, we investigate the secrecy beamforming design under three different levels of FBS-EVE channel state information (CSI), namely, the perfect, imperfect and completely unknown FBS-EVE CSI. Firstly, given the perfect global CSI at the FBS, the PB energy covariance matrix, the FBS information covariance matrix and the time splitting factor are jointly optimized aiming for perfect secrecy rate maximization. Upon assuming the imperfect FBS-EVE CSI, the worst-case and outage-constrained SRM problems corresponding to deterministic and statistical CSI errors are investigated, respectively. Furthermore, considering the more realistic case of unknown FBS-EVE CSI, the artificial noise (AN) aided secrecy beamforming design is studied. Our analysis reveals that for all above cases both the optimal PB energy and FBS information secrecy beamformings are of rank-1. Moreover, for all considered cases of FBS-EVE CSI, the closed-form PB energy beamforming solutions are available when the cross-tier interference constraint is inactive. Numerical simulation results demonstrate the secrecy performance advantages of all proposed secrecy beamforming designs compared to the adopted baseline algorithms.
\end{abstract}

Index Terms-Secrecy beamforming, wireless powered HetNets, worst-case SRM, outage-constrained SRM.

Manuscript received October 18, 2019; revised Feburary 20, 2020; accepted April 25, 2020. The associate editor coordinating the review of this manuscript and approving it for publication was Prof. Besma Smida. This work was supported by the National Natural Science Foundation of China under Grants 61722104, 61671058, and 61620106001, in part by the Sate Key Laboratory of Rail Traffic Control and Safety (Contract No. RCS2018K005), Beijing Jiaotong University, in part by the Science and Technology Development Fund, Macau SAR (File no. 0036/2019/A1 and File no. SKL-IOTSC20182020), and in part by the Research Committee of University of Macau under Grant MYRG2018-00156-FST.

L. Hanzo would like to acknowledge the financial support of the Engineering and Physical Sciences Research Council projects EP/N004558/1, EP/P034284/1, EP/P034284/1, EP/P003990/1 (COALESCE), of the Royal Society's Global Challenges Research Fund Grant as well as of the European Research Council's Advanced Fellow Grant QuantCom.

(Corresponding author: Shaodan Ma.)

S. Gong and C. Xing are with the School of Information and Electronics, Beijing Institute of Technology, Beijing 100081, China. S. Gong was with the Department of Electrical and Computer Engineering, University of Macau. (emails: gsqyxyx@gmail.com, and xingchengwen@gmail.com ).

$\mathrm{S}$. Ma is with the State Key Laboratory of Internet of Things for Smart City and the Department of Electrical and Computer Engineering, University of Macau, Taipa, Macao, China (e-mail: shaodanma@um.edu.mo).

Yonghui $\mathrm{Li}$ is with the School of Electrical and Information Engineering, The University of Sydney, NSW 2006, Australia, and also part time with the Peng Cheng Laboratory, Shenzhen 518055, China. (e-mail: yonghui.li@sydney.edu.au).

Lajos Hanzo is with the School of Electronics and Computer Science, University of Southampton, SO17 1BJ, UK. (e-mail: 1h@ecs.soton.ac.uk).

\section{INTRODUCTION}

With the proliferation of smart devices and data-hungry applications, establishing ubiquitous, high-throughput and secure communications is gaining increased importance in nextgeneration systems [1]-[3]. The traditional macrocells generally have poor performance in terms of indoor coverage and cell edge rate. To tackle this issue, heterogenous networks (HetNets) have emerged as a promising next-generation architecture, which are generally supported by heterogenous base stations (BSs) having different service coverages [4], [5]. Specifically, the macrocell base station (MBS) can provide open access and wide coverage up to dozens of kilometers, while the low-power femtocell base station (FBS) and picocell base station (PBS) are typically deployed in indoor environments and near to femtocell users (FUs) and picocell users (PUs), respectively. As pointed out in [6], the ultradense deployment of femtocells is recognized as an efficient technique to realize 1000 times increase in wireless data rate for. However, due to the high spatial spectrum reuse in HetNets and the dense deployment of FBSs and PBSs, cross-tier interference is usually unavoidable in HetNets. Fortunately, according to [7]-[9], the interference can be reutilized as an effective radio-frequency (RF) energy source for wireless energy harvesting (WEH), which thus contributes to the green and self-sustainable communications. WEH has many advantages over conventional energy supply methods [10]. For example, WEH is more reliable than natural energy supply, such as solar, wind and tide, which are significantly affected by climate and terrain. Also, it is more cost-effective compared to the widely adopted batteries recharge/replacement technique. Generally, the densely deployed HetNets are favorable from the perspective of improving efficiency of WEH, since the distances from energy harvesters to energy stations are substantially shortened.

Recently, WEH-based HetNets have received extension attention, in which the power beacons (PBs) provide power for other nodes via wireless energy transfer [11], [13]-[18]. In [11], H. Tabassum and E. Hossain studied the optimal deployment of PBs in wirelessly powered cellular networks. In [13], the downlink resource allocation problem was investigated by $\mathrm{S}$. Lohani et al. under both time-switching and power-splitting based simultaneous wireless information and power transfer (SWIPT) strategies for two-tier HetNets. The comprehensive analysis of the outage probability and the average ergodic rate in both downlink and uplink stages of wirelessly powered HetNets with different cell associations were presented by S. Akbar et al. in [14]. Y. Zhu et al. in [15] 
further extended the above work into the Massive MIMO aided HetNets with WEH, where different user association schemes are investigated in terms of the achievable average uplink rate. From the view of green communications, the energy efficient beamforming designs for SWIPT HetNets was studied by $\mathbf{M}$. Sheng et al. and H. Zhang et al. in [16] and [17], respectively. To increase energy harvesting efficiency multi-antenna PBs and users, J. Kim et al. in [18] considered sum throughput maximization under different cooperative protocols of two-tier wireless powered cognitive networks.

Furthermore, owing to the open network architectures of HetNets, the security issue faced by wireless powered HetNets has also drawn extensive attention [19]. As a mature technique to guarantee secure communications from the informationtheoretical perspective, physical-layer security (PLS) has been widely researched in both academia and industry [20], [21]. There have been some works considering applying PLS techniques to HetNets with WEH. In [22] and [23], the artificial noise based secrecy rate maximization was studied for secure HetNets with SWIPT. The authors in [24] proposed the maxmin secrecy energy efficiency optimization for wireless powered HetNets, and a distributed ADMM approach was applied to reduce the information exchange overhead. Considering the more practical scenarios where the transmitter only has imperfect eavesdropper's CSI, a secrecy SWIPT strategy for two-tier cognitive radio networks was investigated in [25].

Most of the existing works on HetNets with WEH focus on SWIPT HetNets, it is still a challenging issue that how to design the optimal harvest-then-transmit strategies for HetNets. In this paper, we investigate the secrecy beamforming design for a wirelessly powered HetNet, where the wirelessly powered FBS transmits the confidential information to a single-antenna FU in the presence of a multi-antenna eavesdropper (EVE). The FBS can harvest energy from the PB and the MBS. Moreover, there is no cooperation among the MBS, the PB and the FBS, thus the resultant cross-tier interference is taken into account. In this wirelessly powered HetNet, the energy and information covariance matrices as well as the time splitting factor are jointly optimized to maximize the secrecy rate under different levels of FBS-EVE CSI. Our main contribution is summarized as:

- Firstly, we study the secrecy rate maximization (SRM) problem of wirelessly powered HetNets based on the idealized assumption of perfect global CSI. In order to address this non-convex perfect SRM problem, a relaxed problem using the matrix trace inequality is studied and proved to be tight, since it always provides a rank-1 optimal solution. Considering the joint-convexity and quasiconvexity of the relaxed problem with respect to different variables, a convexity-based line search is proposed for optimally solving the perfect SRM problem. In particular, the closed-form solution of this problem becomes available provided that the cross-tier interference at the MU is negligible.

- Secondly, from a practical viewpoint, we consider the imperfect FBS-EVE CSI scenario with deterministic and Gaussian random CSI errors, respectively. For the deterministic CSI error, the worst-case SRM is studied for guaranteeing the system secrecy performance against the worst-case channel quality, which can be addressed similarly to the perfect SRM problem by using the Sprocedure. As for the Gaussian random CSI error, the outage-constrained SRM problem subject to a probabilistic secrecy constraint is investigated, aiming to ensure system outage secrecy performance widely considered in delay-sensitive or low-latency applications. In this context, the Bernstein-type inequality (BTI) based alternating optimization procedure is proposed. In particular, the rank-1 property of the optimal solutions is validated for both the worst-case and the outage-constrained SRM problems.

- Finally, for the even worse scenario with unknown FBSEVE CSI, we propose applying artificial noise (AN) to improve system secrecy performance. Specifically, our proposed AN aided secrecy beamforming aims to maximize the average AN power subject to the legitimate rate requirement of the FU. This robust design can be reformulated as a concave problem and its optimal rank1 solution is demonstrated. Similarly, the closed-form solution of this AN aided secrecy design is available when the cross-tier interference is negligible.

In fact, the studied SRM problems belong to the nonconvex difference of convex functions (DC) programming, which are more challenging than that in [18] focusing on the sumthroughput maximization of cognitive WPCNs. Compared to the SRM problem of [26] where the single-antenna PB and EVE are assumed, these SRM problems are also more intractable due to the additional energy and interference constraints. Fortunately, we validate that the optimal energy and information beamformings are of rank-1 in the secrecy wirelessly powered HetNet, regardless of the availability of eavesdropper's CSI. This conclusion also provides important insights for practical engineering applications.

Notations: The bold-faced lower-case and upper-case letters stand for vectors and matrices, respectively. The operators $(\cdot)^{\mathrm{T}},(\cdot)^{\mathrm{H}}$ and $(\cdot)^{-1}$ denote the transpose, Hermitian and inverse of a matrix, respectively. $\operatorname{Tr}(\boldsymbol{A})$ and $\operatorname{det}(\boldsymbol{A})$ represent the trace and determinant of $\boldsymbol{A}$, respectively. $\|\cdot\|_{2}$ denotes the matrix spectral norm and $\boldsymbol{A} \succeq \mathbf{0}$ indicates that the square matrix $\boldsymbol{A}$ is positive semidefinite. $\operatorname{rank}(\boldsymbol{A})$ and $\nu(\mathbf{A})$ denote the rank of $\mathbf{A}$ and the unit-norm eigenvector associated with the maximum eigenvalue of $\mathbf{A}$, respectively. Also, $(a)^{+}=$ $\max \{a, 0\}$ is defined. The words 'independent and identically distributed' and 'with respect to' are abbreviated as 'i.i.d.' and 'w.r.t.', respectively.

\section{System MOdEL AND PROBlem Formulation}

As shown in Fig. 1, we consider secure communications of the wirelessly powered HetNet, in which an $N_{M}$-antenna MBS used for information transmission coexists with an $N_{P^{-}}$ antenna PB deployed for wireless energy transfer and an $N_{F^{-}}$ antenna FBS aiming for energy harvesting [27]. Note that the FBS is energy-limited and harvests energy for its communications from the RF signals transmitted by the PB and the MBS. The MBS and the PB transmit the information-bearing signal 


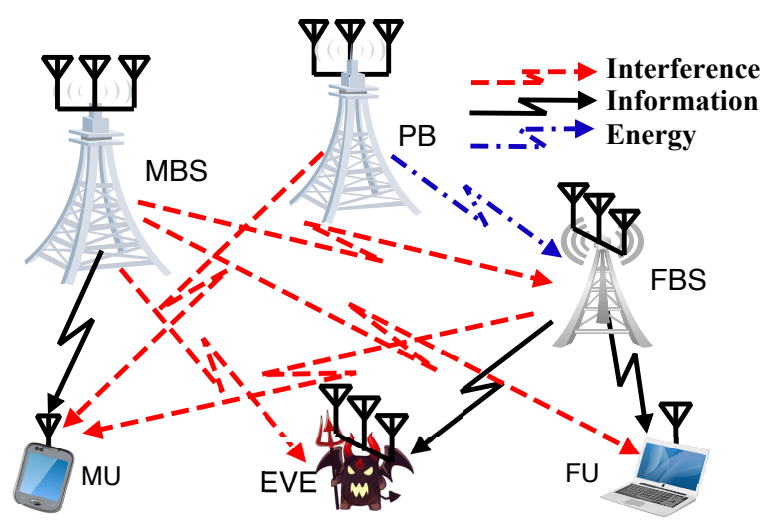

Fig. 1. A secrecy wirelessly powered HetNet.

$\mathbf{s}_{M} \in \mathbb{C}^{N_{M}}$ and energy-bearing signal $\mathbf{s}_{P} \in \mathbb{C}^{N_{P}}$ to a singleantenna MU and an $N_{F}$-antenna FBS, respectively. Then the FBS transmits the confidential signal $\mathbf{s}_{F} \in \mathbb{C}^{N_{F}}$ to a singleantenna FU, while a $N_{E}$-antenna EVE aims for intercepting the signal of the $\mathrm{FBS}^{1}$. We focus our attention on the security of FBS and there is no cooperation between the MBS and the FBS. Hence, the signals transmitted from the MBS and the FBS actually impose interference on the FU and the MU, respectively. All wireless channels are assumed to be quasistatic flat-fading and remain constant during a whole time slot $T$.

In the initial $\tau T$ subslot, where $0<\tau<1$ denotes the time splitting factor, the FBS harvests energy from both the $\mathrm{PB}$ energy signal $\mathbf{s}_{P}$ and MBS interfering signal $\mathbf{s}_{M}$. Let's define $\mathbf{W}_{P}=\mathbb{E}\left[\mathbf{s}_{P} \mathbf{s}_{P}^{H}\right] \in \mathbb{C}^{N_{P} \times N_{P}}$ as the covariance matrix of the PB energy signal $\mathbf{s}_{P}$ subject to the maximum transmit power $P_{P}$, i.e. $\operatorname{tr}\left(\mathbf{W}_{P}\right) \leq P_{P}$, and for simplicity $\mathbb{E}\left[\mathbf{s}_{M} \mathbf{s}_{M}^{H}\right]=$ $\frac{P_{M}}{N_{M}} \mathbf{I}_{N_{M}}$ with $P_{M}$ being the MBS maximum transmit power. By neglecting the contribution of thermal noise to the total harvested energy at the FBS, the amount of energy harvested at the FBS is expressed as

$$
E\left(\mathbf{W}_{P}\right)=\tau T \xi\left(\operatorname{tr}\left(\mathbf{H}_{F} \mathbf{W}_{P} \mathbf{H}_{F}^{H}\right)+\frac{P_{M}}{N_{M}} \operatorname{tr}\left(\mathbf{G}_{F} \mathbf{G}_{F}^{H}\right)\right)
$$

where $0<\xi<1$ is the energy harvesting efficiency factor. $\mathbf{H}_{F} \in \mathbb{C}^{N_{F} \times N_{P}}$ and $\mathbf{G}_{F} \in \mathbb{C}^{N_{F} \times N_{M}}$ denote the PB-FBS channel and MBS-FBS channel, respectively. Next, in the second $(1-\tau) T$ subslot, the FBS transmits the confidential signal $\mathbf{s}_{F}$ to the FU by utilizing the harvested energy in (1). The signals received at the FU and the EVE are then expressed as

$$
\begin{aligned}
& y_{R}=\mathbf{h}_{R}^{H} \mathbf{s}_{F}+\mathbf{g}_{R}^{H} \mathbf{s}_{M}+n_{R}, \\
& \mathbf{y}_{E}=\mathbf{H}_{E} \mathbf{s}_{F}+\mathbf{G}_{E} \mathbf{s}_{M}+\mathbf{n}_{E},
\end{aligned}
$$

where $\mathbf{h}_{R} \in \mathbb{C}^{N_{F}}$ and $\mathbf{g}_{R} \in \mathbb{C}^{N_{M}}$ denote the FBS-FU channel and the MBS-FU channel, respectively. $\mathbf{H}_{E} \in \mathbb{C}^{N_{E} \times N_{F}}$ and $\mathbf{G}_{E} \in \mathbb{C}^{N_{E} \times N_{M}}$ denote the FBS-EVE channel and the MBS-EVE channel, respectively. $n_{R} \sim \mathcal{C N}\left(0, \sigma_{n}^{2}\right)$ and

\footnotetext{
${ }^{1}$ In contrast to our previous study of wireless powered downlink secure communications of the FBS, the uplink secure transmissions from the FU to the FBS essentially belong to the classical MIMO multi-antenna eavesdropper (MIMOME) wiretap scenario, which has been widely researched in the open literature [21].
}

$\mathbf{n}_{E} \sim \mathcal{C N}\left(\mathbf{0}, \sigma_{n}^{2} \mathbf{I}_{N_{E}}\right)$ are i.i.d circularly symmetric Gaussian noises at the FU and the EVE, respectively. Additionally, we define $\mathbf{P}_{F}=\mathbb{E}\left[\mathbf{s}_{F} \mathbf{s}_{F}^{H}\right] \in \mathbb{C}^{N_{F} \times N_{F}}$ as the covariance matrix of the FBS information signal $\mathbf{s}_{F}$, the achievable rates (in $\mathrm{bps} / \mathrm{Hz}$ ) at the $\mathrm{FU}$ and the EVE are then given by

$$
\begin{aligned}
R_{I} & =(1-\tau) \log _{2}\left(1+\frac{\mathbf{h}_{R}^{H} \mathbf{P}_{F} \mathbf{h}_{R}}{\sigma_{n}^{2}+\frac{P_{M}}{N_{M}}\left\|\mathbf{g}_{R}\right\|^{2}}\right), \\
R_{E} & =(1-\tau) \log _{2} \operatorname{det}\left(\mathbf{I}_{N_{E}}+\left(\sigma_{n}^{2} \mathbf{I}_{N_{E}}\right.\right. \\
& \left.\left.+\frac{P_{M}}{N_{M}} \mathbf{G}_{E} \mathbf{G}_{E}^{H}\right)^{-1} \mathbf{H}_{E} \mathbf{P}_{F} \mathbf{H}_{E}^{H}\right) .
\end{aligned}
$$

According to [22], the achievable secrecy rate $R_{S}$ of the wirelessly powered HetNet is actually the data rate at which the desired information is correctly decoded by the FU, while no information is wiretapped by the EVE. Mathematically, we have

$$
R_{S}=\left[R_{I}-R_{E}\right]^{+} .
$$

In our work, we jointly optimize the PB and FBS transmit covariance matrices $\left\{\mathbf{W}_{P}, \mathbf{P}_{F}\right\}$ and the time splitting factor $\tau$ for maximizing the achievable secrecy rate $R_{S}$ in (4). The resultant SRM problem for the wirelessly powered HetNet is then formulated as

$R_{S}^{\star}=\max _{\tau, \mathbf{W}_{P} \succeq \mathbf{0}, \mathbf{P}_{F} \succeq \mathbf{0}} R_{I}-R_{E}$

s.t. $\mathrm{CR} 1: 0 \leq \tau \leq 1, \operatorname{tr}\left(\mathbf{W}_{P}\right) \leq P_{P}$

CR2: $(1-\tau) \operatorname{tr}\left(\mathbf{P}_{F}\right) \leq \tau \xi\left[\operatorname{tr}\left(\mathbf{H}_{F} \mathbf{W}_{P} \mathbf{H}_{F}^{H}\right)+\frac{P_{M}}{N_{M}} \operatorname{tr}\left(\mathbf{G}_{F} \mathbf{G}_{F}^{H}\right)\right]$,

CR3: $\tau \mathbf{h}_{M}^{H} \mathbf{W}_{P} \mathbf{h}_{M}+(1-\tau) \mathbf{g}_{p}^{H} \mathbf{P}_{F} \mathbf{g}_{p} \leq I_{t h}$.

In problem (5), the constraint CR1 comes from the fact that the PB transmit power has a maximum threshold, and CR2 denotes the energy causality constraint of the wirelessly powered FBS. While CR3 models the average interference constraint of the secrecy wirelessly powered HetNet. Specifically, by defining $\mathbf{h}_{M} \in \mathbb{C}^{N_{P}}$ and $\mathbf{g}_{p} \in \mathbb{C}^{N_{F}}$ as the PB-MU channel and the FBS-MU channel, respectively, the terms $\mathbf{h}_{M}^{H} \mathbf{W}_{P} \mathbf{h}_{M}$ and $\mathbf{g}_{p}^{H} \mathbf{P}_{F} \mathbf{g}_{p}$ actually denote the total interference at the MU originating from the PB and the FBS, respectively. Since the PB energy transfer and the FBS information transfer are separated by the time splitting factor $\tau$, we consider the average interference power constraint at the MU as shown in $\mathrm{CR} 3$, where $I_{t h}$ denotes the minimum tolerable interference. It is readily inferred from CR3 that problem (5) is feasible for an arbitrary $I_{t h} \geq 0$. However, due to the highly coupled variables $\left\{\tau, \mathbf{W}_{P}, \mathbf{P}_{F}\right\}$, the SRM problem (5) is generally non-convex and challenging to address.

In the sequel, we will investigate the SRM under three different levels of the FBS-EVE CSI. In the first case, the global CSI of the wirelessly powered HetNet is available at the FBS via channel feedback and high-SNR training techniques. In the second case, by assuming imperfect FBS-EVE CSI at the FBS, a pair of robust SRM problems are investigated under deterministic and Gaussian random CSI errors, respectively. In the third case, we consider the more practical scenario that the FBS is not aware of the existence of EVE. In other words, the FBS-EVE CSI is completely unknown to the FBS. 


\section{PERFECT SRM UNDER GLOBAL CSI OF THE WIRELESSLY POWERED HETNET}

In this section, a convexity-based one dimensional search is proposed for optimally solving the SRM problem (5) under the global CSI of the wirelessly powered HetNet.

A. Transformation of Problem (5)

Firstly, by introducing an auxiliary variable $\eta$, the SRM problem (5) can be reformulated as

$$
\begin{aligned}
& R_{S}^{\star}=\max _{\tau, \mathbf{W}_{P} \succeq \mathbf{0}, \mathbf{P}_{F} \succeq \mathbf{0}, \eta}(1-\tau) \log _{2}\left(1+C_{1} \mathbf{h}_{R}^{H} \mathbf{P}_{F} \mathbf{h}_{R}\right) \\
&-(1-\tau) \log _{2} \eta
\end{aligned}
$$

s.t. $\mathrm{CR} 1 \sim \mathrm{CR} 3, \mathrm{CR} 4: \operatorname{det}\left(\mathbf{I}_{N_{E}}+\mathbf{R}_{E} \mathbf{H}_{E} \mathbf{P}_{F} \mathbf{H}_{E}^{H}\right) \leq \eta$,

where $C_{1}=\left(\sigma_{n}^{2}+\frac{P_{M}}{N_{M}}\left\|\mathbf{g}_{R}\right\|^{2}\right)^{-1}$ and $\mathbf{R}_{E}=\left(\sigma_{n}^{2} \mathbf{I}_{N_{E}}+\right.$ $\left.\frac{P_{M}}{N_{M}} \mathbf{G}_{E} \mathbf{G}_{E}^{H}\right)^{-1}$. Unfortunately, problem (6) is still difficult to address because of the nonconvex constraint CR4. In order to tackle this issue, we firstly consider a relaxation of CR4 based on the following lemma.

Lemma 1. [29] For any positive semi-definite matrix $\mathbf{A} \succeq \mathbf{0}$, we have $\operatorname{det}(\mathbf{I}+\mathbf{A}) \geq 1+\operatorname{tr}(\mathbf{A})$, where the equality holds if and only if $\operatorname{rank}(\mathbf{A})=1$.

By applying Lemma 1 to CR4, problem (6) is relaxed to

$$
\begin{gathered}
\tilde{R}_{S}^{\star}=\max _{\substack{\tau, \mathbf{W}_{P} \succeq \mathbf{0}, \mathbf{P}_{F} \succeq \mathbf{0}, \eta}}(1-\tau) \log _{2}\left(1+C_{1} \mathbf{h}_{R}^{H} \mathbf{P}_{F} \mathbf{h}_{R}\right)-(1-\tau) \log _{2} \eta \\
\text { s.t. } \quad \text { CR1 } \sim \text { CR3, CR4: } 1+\operatorname{tr}\left(\mathbf{R}_{E} \mathbf{H}_{E} \mathbf{P}_{F} \mathbf{H}_{E}^{H}\right) \leq \eta, \quad(7)
\end{gathered}
$$

where $\tilde{R}_{S}^{\star}$ denotes the achievable maximum secrecy rate by solving problem (7). Based on Lemma 1, problem (7) clearly has a larger feasible region than problem (6), so that $\tilde{R}_{S}^{\star} \geq R_{S}^{\star}$ holds. We then introduce new variables $\widetilde{\mathbf{W}}_{P}=\tau \mathbf{W}_{P}$ and $\widetilde{\mathbf{P}}_{F}=(1-\tau) \mathbf{P}_{F}$ to equivalently transform problem (7) into

$$
\begin{aligned}
& \tilde{R}_{S}^{\star}= \max _{\tau, \widetilde{\mathbf{W}}_{P} \succeq \mathbf{0}, \widetilde{\mathbf{P}}_{F} \succeq \mathbf{0}, \eta}(1-\tau) \log _{2}\left(\frac{1+\frac{C_{1} \mathbf{h}_{R}^{H} \widetilde{\mathbf{P}}_{F} \mathbf{h}_{R}}{1-\tau}}{\eta}\right) \\
&\text { s.t. } \left.\widetilde{\mathrm{CR} 1}: 0 \leq \tau \leq 1, \operatorname{tr}\left(\widetilde{\mathbf{W}}_{P}\right) \leq \tau P_{P},\right) \\
& \widetilde{\mathrm{CR} 2}: \operatorname{tr}\left(\widetilde{\mathbf{P}}_{F}\right) \leq \xi \operatorname{tr}\left(\mathbf{H}_{F} \widetilde{\mathbf{W}_{P}} \mathbf{H}_{F}^{H}\right)+\xi \tau \frac{P_{M}}{N_{M}} \operatorname{tr}\left(\mathbf{G}_{F} \mathbf{G}_{F}^{H}\right), \\
&\left.\widetilde{\mathrm{CR} 3}: \mathbf{h}_{M}^{H} \widetilde{\mathbf{W}} \mathbf{h}_{M}+\mathbf{g}_{p}^{H} \widetilde{\mathbf{P}}_{F} \mathbf{g}_{p} \leq I_{t h},\right) \\
& \widetilde{\mathrm{CR} 4}: 1-\tau+\operatorname{tr}\left(\mathbf{R}_{E} \mathbf{H}_{E} \widetilde{\mathbf{P}}_{F} \mathbf{H}_{E}^{H}\right) \leq \eta(1-\tau) .
\end{aligned}
$$

It is concluded from problem (8) that for any fixed $\eta$ the objective function is the perspective of a strictly concave matrix function $f\left(\widetilde{\mathbf{P}}_{F}\right)=\log _{2}\left(\frac{1+C_{1} \mathbf{h}_{R}^{H} \widetilde{\mathbf{P}}_{F} \mathbf{h}_{R}}{\eta}\right)$, which is also strictly concave [30, p. 39]. Moreover, all constraints in problem (8) are convex. Therefore, it is inferred that problem (8) is jointly concave w.r.t. $\left\{\tau, \widetilde{\mathbf{W}}_{P}, \widetilde{\mathbf{P}}_{F}\right\}$ given any $\eta$, and can be globally solved by the interior point method. To further investigate the tightness of the constraint $\widetilde{\mathrm{CR} 3}$ when varying $I_{t h}$, we firstly consider solving problem (8) without $\widetilde{\mathrm{CR} 3}$

$$
\begin{aligned}
& \max _{\tau, \widetilde{\mathbf{W}}_{P} \succeq \mathbf{0}, \widetilde{\mathbf{P}}_{F} \succeq \mathbf{0}, \eta}(1-\tau) \log _{2}\left(\frac{1+\frac{C_{1} \mathbf{h}_{R}^{H} \widetilde{\mathbf{P}}_{F} \mathbf{h}_{R}}{1-\tau}}{\eta}\right) \\
& \text { s.t. } \widetilde{\mathrm{CR} 1}, \widetilde{\mathrm{CR} 2}, \widetilde{\mathrm{CR} 4} .
\end{aligned}
$$

Let's define $\widetilde{\mathbf{W}}_{P, I_{t h}}$ and $\widetilde{\mathbf{P}}_{F, I_{t h}}$ as the optimal solution to problem (9). The corresponding interference level is then expressed as $\tilde{I}_{t h}=\mathbf{h}_{M}^{H} \widehat{\mathbf{W}}_{P, I_{t h}} \mathbf{h}_{M}+\mathbf{g}_{p}^{H} \widetilde{\mathbf{P}}_{F, I_{t h}} \mathbf{g}_{p}$. When $I_{t h}>\tilde{I}_{t h}$, it is readily inferred that the constraint $\widetilde{\mathrm{CR} 3}$ in problem (8) will be automatically satisfied using the optimal solution $\left\{\widetilde{\mathbf{W}}_{P, I_{t h}}, \widetilde{\mathbf{P}}_{F, I_{t h}}\right\}$ to problem (9), implying that in this context $\widehat{\mathrm{CR} 3}$ has no effect on problem (8) and can be neglected without loss of optimality. While for the case of $0 \leq I_{t h} \leq \tilde{I}_{t h}$, we provide an interesting insight in the following Theorem. Theorem 1. When $0 \leq I_{t h} \leq \tilde{I}_{t h}$, the constraint CR3 in problem (8) is tight, which implies that the optimal solution to problem (8) lies at the boundary of $\mathbf{h}_{M}^{H} \widetilde{\mathbf{W}}_{P} \mathbf{h}_{M}+\mathbf{g}_{p}^{H} \widetilde{\mathbf{P}}_{F} \mathbf{g}_{p}=$ $I_{t h}$.

Proof. Please refer to Appendix A-A.

Since the globally optimal solution $\left\{\tau, \widetilde{\mathbf{W}}_{P}, \widetilde{\mathbf{P}}_{F}\right\}$ to problem (8) for any fixed $\eta$ is available, our remaining task is to find the globally optimal $\eta$, as presented in Theorem 2 .

Theorem 2. Problem (8) is quasi-concave w.r.t. $\eta$, and the globally optimal $\eta$ can be computed by a one-dimensional search, i.e., Golden section search (GSS) [30].

Proof. Let's rewrite problem (8) by introducing an auxiliary variable $t$ as

$$
\begin{aligned}
& \underset{\tau, \eta, t, \widetilde{\mathbf{W}}_{P} \succeq \mathbf{0}, \widetilde{\mathbf{P}}_{F} \succeq \mathbf{0}}{\max }(1-\tau) \log _{2}\left(1+\frac{C_{1} \mathbf{h}_{R}^{H} \widetilde{\mathbf{P}}_{F} \mathbf{h}_{R}}{1-\tau}\right)+t \\
& \text { s.t. } \widetilde{\mathrm{CR} 1} \sim \widetilde{\mathrm{CR} 3}, \\
& \widetilde{\mathrm{CR} 4}: 1-\tau+\operatorname{tr}\left(\mathbf{R}_{E} \mathbf{H}_{E} \widetilde{\mathbf{P}}_{F} \mathbf{H}_{E}^{H}\right) \leq \eta(1-\tau) \\
& \widetilde{\mathrm{CR} 5}: t \leq(1-\tau) \log _{2} \frac{1}{\eta} .
\end{aligned}
$$

Given any $\eta$, problem (10) is jointly and strictly concave w.r.t. $\left\{\tau, t, \widetilde{\mathbf{W}}_{P}, \widetilde{\mathbf{P}}_{F}\right\}$ and thus the unqiue optimal solution exists. Based on this, it is readily inferred that the value of (10a) is continuous on $\eta$. We observe that for a sufficiently small $\eta$, the value of (10a) is strongly dominated by the active constraint (10c). Upon increasing $\eta$, the feasible region specified by (10c) expands and thus the value of (10a) increases. However, when $\eta$ becomes large enough, the constraint (10d) with the small $\log _{2} \frac{1}{\eta}$ actually dominates the value of (10a). In this context, we find that the value of (10a) decreases with increasing $\eta$. According to the above analysis, it can be inferred that there must exist a turning point $\hat{\eta}$ for problem (8). Specifically, with the increase of small $\eta$, the value of (10a) firstly increases until $\eta$ reaches $\hat{\eta}$, and then decreases. This property hints that problem (8) is unimodal (quasi-concave) w.r.t. $\eta$, of which the globally optimal value can be found by GSS.

According to Theorem 2, we firstly determine the onedimensional search interval of $\eta$ as follows. For achieving a nonzero secrecy rate, the maximum value of $\eta$ actually corresponds to the maximum legitimate rate $R_{u p}$ of the FU, which is derived by solving the following problem

$$
R_{u p}=\max _{\tau, \widetilde{\mathbf{W}}_{P} \succeq \mathbf{0}, \widetilde{\mathbf{P}}_{F} \succeq \mathbf{0}}(1-\tau) \log _{2}\left(1+\frac{C_{1} \mathbf{h}_{R}^{H} \widetilde{\mathbf{P}}_{F} \mathbf{h}_{R}}{1-\tau}\right)
$$




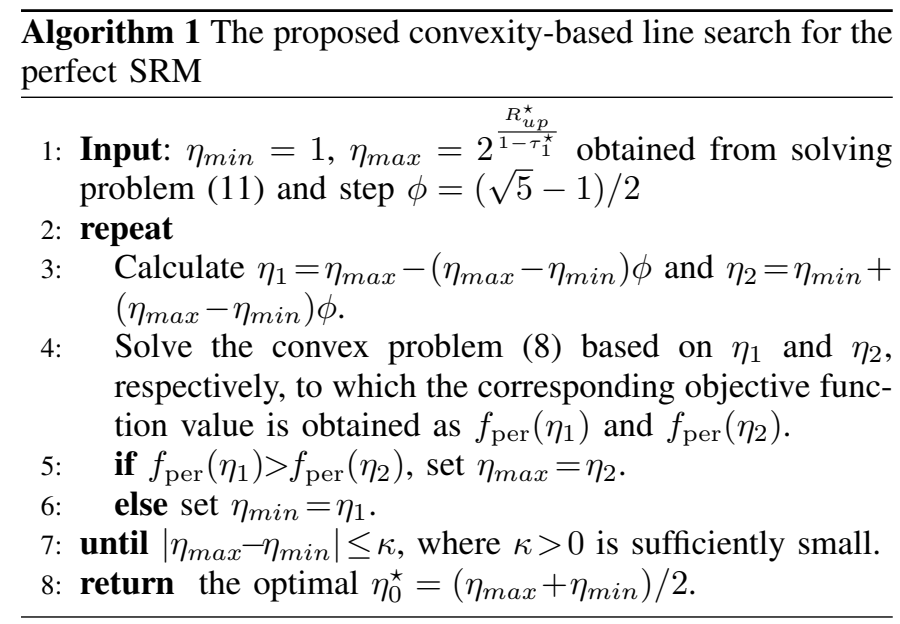

It is clear that problem (11) is also jointly concave w.r.t. $\left\{\tau, \widetilde{\mathbf{W}}_{P}, \widetilde{\mathbf{P}}_{F}\right\}$. With the optimal solution $\left\{R_{u p}^{\star}, \tau_{1}^{\star}\right\}$ to problem (11), we readily infer that the value range of $\eta$ is $1 \leq \eta \leq 2^{\frac{R_{u p}^{\star}}{1-\tau_{1}^{\star}}}$. Overall, given any $\eta$, by combining the joint concavity of problem (8) with the GSS for finding the globally-optimal $\eta$, problem (8) can be optimally solved. More importantly, we further prove that $R_{S}^{\star}=\tilde{R}_{S}^{\star}$ holds for the PSRM problem (5) and the relaxed problem (8), as shown in Theorem 3.

Theorem 3. Problem (8) is a tight relaxation of the perfect SRM problem (5). In other words, the optimal solution $\left\{\tau^{\star}, \eta^{\star}, \widetilde{\mathbf{W}}_{P}^{\star}, \widetilde{\mathbf{P}}_{F}^{\star}\right\}$ to problem (8) satisfies $\operatorname{rank}\left(\widetilde{\mathbf{W}}_{P}^{\star}\right)=$ $\operatorname{rank}\left(\widetilde{\mathbf{P}}_{F}^{\star}\right)=1$ and thus $\tilde{R}_{S}^{\star}=R_{S}^{\star}$ holds. By recalling $\widetilde{\mathbf{W}}_{P}=$ $\tau \mathbf{W}_{P}$ and $\widetilde{\mathbf{P}}_{F}=(1-\tau) \mathbf{P}_{F}$, the corresponding $\left\{\tau^{\star}, \mathbf{W}_{P}^{\star}, \mathbf{P}_{F}^{\star}\right\}$ are also globally-optimal to problem (5).

Proof. Please refer to Appendix A-B.

Following the proof of Theorem 3, we propose a convexitybased line search for globally solving the perfect SRM problem (5). To be specific, given any $\eta$, the relaxed convex problem (8) is firstly addressed for obtaining the optimal solution $\left\{\tau^{\star}, \mathbf{W}_{P}^{\star}, \mathbf{P}_{F}^{\star}\right\}$ (via variable substitution) and the resultant achievable secrecy rate. Then the GSS is applied to find the globally-optimal $\eta^{\star}$. The detailed algorithm is summarized in Algorithm 1. Theorem 3 further reveals that the optimal energy and information covariance matrices, i.e. $\mathbf{W}_{P}^{\star}$ and $\mathbf{P}_{F}^{\star}$, to problem (5) satisfy $\operatorname{rank}\left(\mathbf{W}_{P}^{\star}\right)=\operatorname{rank}\left(\mathbf{P}_{F}^{\star}\right)=1$. Physically, this property means that single-stream transmission of both PB and FBS are optimal for secrecy performance of the wirelessly powered HetNet.

\section{RoBUST SRMS UNDER IMPERFECT FBS-EVE CSI}

In this section, two types of robust SRM problems are investigated in depth for the secrecy wirelessly powered HetNet. Specifically, one is the worst-case SRM associated with deterministic FBS-EVE CSI error. In this case, we propose a convexity-based line search method for finding the optimal worst-case solution. The other is the outage-constrained SRM subject to Gaussian random FBS-EVE CSI error, for which the convex reformulation is realized by introducing an auxiliary variable.

\section{A. The Proposed Worst-Case SRM}

Firstly, for the deterministically imperfect FBS-EVE channel, implying that the EVE is a legitimate but unintended user in our system, and the feedback of the FBS-EVE channel to the FBS suffers from certain bounded estimation or quantization error [26], [29], we have $\mathbf{H}_{E}=\widehat{\mathbf{H}}_{E}+\Delta \mathbf{H}_{E}$, where $\widehat{\mathbf{H}}_{E_{k}}$ denotes the estimated FBS-EVE channel, while $\Delta \mathbf{H}_{E}$ is the norm bounded CSI error satisfying $\left\|\Delta \mathbf{H}_{E}\right\|_{F} \leq \xi_{f}$. Based on this, the achievable worst-case secrecy rate of the wirelessly powered HetNet is given by $R_{S, R o}=R_{I}-\max _{\Delta \mathbf{H}_{E}} R_{E}$, and the resultant worst-case SRM problem is formulated as

$$
R_{S, R o}^{\star}=\max _{\substack{\tau, \mathbf{W}_{P} \succeq \mathbf{0} \\ \mathbf{P}_{F} \succeq \mathbf{0}}} \min _{\Delta \mathbf{H}_{E}} R_{I}-R_{E}, \quad \text { s.t. } \mathrm{CR} 1 \sim \mathrm{CR} 3 .
$$

As with the reformulation of perfect SRM problem (5), we also introduce the auxiliary variable $\eta$ to reformulate problem (12) as

$$
R_{S, R o}^{\star}=\max _{\substack{\tau, \mathbf{W}_{P} \succeq \mathbf{0}, \mathbf{P}_{F} \succeq \mathbf{0}, \eta}}(1-\tau) \log _{2}\left(1+C_{1} \mathbf{h}_{R}^{H} \mathbf{P}_{F} \mathbf{h}_{R}\right)-(1-\tau) \log _{2} \eta
$$$$
\text { s.t. } \quad \mathrm{CR} 1 \sim \mathrm{CR} 3 \text {, }
$$$$
\text { CR5: } \operatorname{det}\left(\mathbf{I}_{N_{E}}+\boldsymbol{R}_{E} \mathbf{H}_{E} \mathbf{P}_{F} \mathbf{H}_{E}^{H}\right) \leq \eta,\left\|\Delta \mathbf{H}_{E}\right\|_{F} \leq \xi_{f} \text {. }
$$

Compared to the perfect SRM problem (5), problem (13) is more challenging since the semi-infinite norm bounded CSI error is included in CR5. To make problem (13) tractable, we firstly relax it using the above Lemma 1 to

$$
\tilde{R}_{S, R o}^{\star}=\max _{\substack{\tau, \mathbf{W}_{P} \succeq \mathbf{0}, \mathbf{P}_{F} \succeq \mathbf{0}, \eta}}(1-\tau) \log _{2}\left(1+C_{1} \mathbf{h}_{R}^{H} \mathbf{P}_{F} \mathbf{h}_{R}\right)-(1-\tau) \log _{2} \eta
$$

s.t. $\quad \mathrm{CR} 1 \sim \mathrm{CR} 3$,

CR5: $1+\operatorname{tr}\left(\mathbf{R}_{E} \mathbf{H}_{E} \mathbf{P}_{F} \mathbf{H}_{E}^{H}\right) \leq \eta,\left\|\Delta \mathbf{H}_{E}\right\|_{F} \leq \xi_{f}$,

where $\tilde{R}_{S, R o}^{\star}$ denotes the maximum achievable worst-case secrecy rate by solving problem (14) and satisfies $\tilde{R}_{S, R o}^{\star} \geq$ $R_{S, R o}^{\star}$ due to larger feasible region of problem (14). For solving this non-convex problem effectively, both the equality $\mathbf{h}_{E}=\operatorname{vec}\left(\left(\hat{\mathbf{H}}_{E}+\Delta \mathbf{H}_{E}\right)^{H}\right)=\hat{\mathbf{h}}_{E}+\boldsymbol{\Delta} \mathbf{h}_{E}$ and the identity $\operatorname{Tr}\left(\mathbf{A}^{H} \mathbf{B C D}\right)=\operatorname{vec}(\mathbf{A})^{H}\left(\mathbf{D}^{T} \otimes \mathbf{B}\right) \operatorname{vec}(\mathbf{C})$ are utilized to rewrite the constraint $\mathrm{CR} 5$ as

$1+\left(\hat{\mathbf{h}}_{E}+\Delta \mathbf{h}_{E}\right)^{H}\left(\mathbf{R}_{E} \otimes \mathbf{P}_{F}\right)\left(\hat{\mathbf{h}}_{E}+\Delta \mathbf{h}_{E}\right) \leq \eta,\left\|\Delta \mathbf{h}_{E}\right\|_{F} \leq \zeta_{f}$.

Lemma 2. (S-procedure) [31] For the equation $f(\mathbf{x})=$ $\mathbf{x}^{H} \mathbf{A} \mathbf{x}+\mathbf{x}^{H} \mathbf{b}+\mathbf{b}^{H} \mathbf{x}+c$, in which $\mathbf{x} \in \mathbb{C}^{N}, \mathbf{A} \in \mathbb{H}^{N \times N}$, $\mathbf{b} \in \mathbb{C}^{N}$ and $c$ is a constant, the following equality holds

$$
\begin{aligned}
& f(\mathbf{x}) \leq 0, \forall \mathbf{x} \in\left\{\mathbf{x} \mid \operatorname{tr}\left(\mathbf{x} \mathbf{x}^{H}\right) \leq \epsilon_{e}\right\} \\
& \Leftrightarrow u\left[\begin{array}{cc}
\mathbf{I}_{N} & \mathbf{0}_{N \times 1} \\
\mathbf{0}_{N \times 1}^{T} & \epsilon_{e}
\end{array}\right]-\left[\begin{array}{cc}
\mathbf{A} & \mathbf{b} \\
\mathbf{b}^{H} & c
\end{array}\right] \succeq \mathbf{0}, \text { with some } u \geq 0 .
\end{aligned}
$$

We then take advantage of Lemma 2 and auxiliary variables $\widetilde{\mathbf{W}}_{P}=\tau \mathbf{W}_{P}, \widetilde{\mathbf{P}}_{F}=(1-\tau) \mathbf{P}_{F}$ to transform the nonlinear semi-infinite constraint (15) into a LMI. Then the relaxed WSRM problem (14) is re-expressed as problem (17) (shown at the top of next page), where a scalar variable $u>0$ is introduced. In contrast to problem (8), an additional SDP 


$$
\begin{aligned}
& \tilde{R}_{S, R o}^{\star}=\max _{\tau, u, \eta, \widetilde{\mathbf{W}}_{P} \succeq \mathbf{0}, \widetilde{\mathbf{P}}_{F} \succeq \mathbf{0}}(1-\tau) \log _{2}\left(1+\frac{C_{1} \mathbf{h}_{R}^{H} \widetilde{\mathbf{P}}_{F} \mathbf{h}_{R}}{1-\tau}\right)-(1-\tau) \log _{2} \eta \\
& \text { s.t. } \widetilde{\mathrm{CR} 1} \sim \widetilde{\mathrm{CR} 3}, \widetilde{\mathrm{CR} 5}:\left[\begin{array}{cc}
u \mathbf{I}_{N}-\left(\mathbf{R}_{E}^{T} \otimes \widetilde{\mathbf{P}}_{F}\right) & -\left(\mathbf{R}_{E}^{T} \otimes \widetilde{\mathbf{P}}_{F}\right) \hat{\mathbf{h}}_{E} \\
-\hat{\mathbf{h}}_{E}^{H}\left(\mathbf{R}_{E}^{T} \otimes \widetilde{\mathbf{P}}_{F}\right) & (\eta-1)(1-\tau)-u \zeta_{f}^{2}-\hat{\mathbf{h}}_{E}^{H}\left(\mathbf{R}_{E}^{T} \otimes \widetilde{\mathbf{P}}_{F}\right) \hat{\mathbf{h}}_{E}
\end{array}\right] \succeq \mathbf{0},
\end{aligned}
$$

constraint $\widetilde{\mathrm{CR} 5}$ is included in problem (17). For any fixed $\eta$, we easily find that $\widetilde{\mathrm{CR} 5}$ is a convex linear matrix inequality (LMI) w.r.t. $\left\{\tau, u, \widetilde{\mathbf{P}}_{F}\right\}$, so problem (17) becomes jointly concave w.r.t. $\left\{\tau, u, \widetilde{\mathbf{W}}_{P}, \widetilde{\mathbf{P}}_{F}\right\}$. Similar to Theorem 2, we can also prove that problem (17) is quasi-concave w.r.t $\eta$, since the semi-infinite CSI error $\Delta \mathbf{h}_{E}$ in the equivalent problem (14) is independent of $\eta$. Based on the above discussions, it is readily inferred that the proposed convexity-based line search for problem (8) can be directly extended to problem (17). More importantly, an interesting insight is provided in the following Theorem, namely, problem (17) also admits the rank-1 optimal solution.

Theorem 4. Problem (17) is a tight relaxation of the worstcase SRM problem (12), which means that its optimal solution $\left\{\tau^{\star}, \eta^{\star}, \widetilde{\mathbf{W}}_{P}^{\star}, \widetilde{\mathbf{P}}_{F}^{\star}\right\}$ satisfies $\operatorname{rank}\left(\widetilde{\mathbf{W}}_{P}^{\star}\right)=\operatorname{rank}\left(\widetilde{\mathbf{P}}_{F}^{\star}\right)=1$ and $\tilde{R}_{S, R o}^{\star}=R_{S, R o}^{\star}$. Meanwhile, the corresponding original variables $\left\{\tau^{\star}, \mathbf{W}_{P}^{\star}, \mathbf{P}_{F}^{\star}\right\}$ are optimal for problem (12).

Proof. Please refer to Appendix C.

Generally, the proof of Theorem 4 subject to the complicated LMI constraint CR5 is more difficult than that of Theorem 3. Based on Theorem 4, the globally optimal solution $\left\{\tau^{\star}, \mathbf{W}_{P}^{\star}, \mathbf{P}_{F}^{\star}\right\}$ to the worst-case SRM problem (12) can also be obtained by successively solving the relaxed problem (17) for which the proposed convexity-based line search in Algorithm 1 still works.

\section{B. The Proposed Outage-Constrained SRM}

It is widely recognized that the worst-case optimization represents the most conservative robust design, which is only encountered in practical systems with a low probability [28], [32]. Hence, in this subsection, we consider the more general case of statistically imperfect CSI, in which the FBS-EVE CSI error is assumed to be complex Gaussian distributed, i.e., $\Delta \mathbf{h}_{E}=\operatorname{vec}\left(\Delta \mathbf{H}_{E}^{H}\right) \sim \mathcal{C N}\left(\mathbf{0}, \mathbf{C}_{E}\right)$, where $\mathbf{C}_{E} \in \mathbb{C}^{N_{F} N_{E} \times N_{F} N_{E}}$ denotes the positive semi-definite error covariance matrix. Inspired by the fact that under the unbounded Gaussian error $\Delta \mathbf{h}_{E}$, an absolutely safe beamforming design cannot be guaranteed, we instead consider the outageconstrained SRM to implement secure communications in the wirelessly powered HetNet. More specifically, by defining the maximum secrecy rate outage probability $p_{\text {out }}$, a $100\left(1-p_{\text {out }}\right) \%$-safe design of our wirelessly powered HetNet is investigated. Mathematically, the outage-constrained SRM problem is formulated as

$$
\begin{aligned}
& \max _{\tau_{0}, \mathbf{W}_{P} \succeq \mathbf{0}, \mathbf{P}_{F} \succeq \mathbf{0}} R_{S} \\
& \text { s.t. } \quad \mathrm{CR} 1 \sim \mathrm{CR} 3, \\
& \text { CR6 : } \operatorname{Pr}_{\Delta \mathbf{H}_{E}}\left\{R_{I}-R_{E} \geq R_{S}\right\} \geq 1-\text { pout }_{\text {out }} .
\end{aligned}
$$

Clearly, the secrecy outage constraint CR6 indicates that the probability of the achievable secrecy rate being over $R_{S}$ should be higher than $1-p_{\text {out }}$, and problem (18) aims for maximizing this $100 p_{\text {out }} \%$-outage secrecy rate threshold $R_{S}$. However, problem (18) is computationally intractable since the constraint CR6 does not have an explicit expression. Therefore, we consider replacing the function $R_{I}-R_{E}$ in CR6 by an easy-to-handle function via the following Lemmas.

Lemma 3. [33] For an arbitrary positive-definite matrix $\mathbf{E} \in$ $\mathbb{C}^{N \times N}$, we have $-\ln \operatorname{det}(\mathbf{E})=\underset{\mathbf{S} \succeq \mathbf{0}}{\max }-\operatorname{tr}(\mathbf{S E})+\ln \operatorname{det}(\mathbf{S})+N$, where the optimal $\mathbf{S}^{\star}$ is derived as $\mathbf{S}^{\star}=\mathbf{E}^{-1}$.

Lemma 4. (Bernstein-type inequality (BTI) [28]) For an arbitrary vector $\mathbf{x} \in \mathcal{C N}(\mathbf{0}, \mathbf{I})$, we assume $f(\mathbf{x})=\mathbf{x}^{H} \mathbf{A x}+$ $2 \operatorname{Re}\left\{\mathbf{x}^{H} \mathbf{b}\right\}+c$, where $\mathbf{A} \in \mathbb{H}^{N \times N}, \mathbf{b} \in \mathbb{C}^{N}$ and $c$ is a constant. Then for any $p_{\text {out }} \in[0,1]$, the following convex approximation holds, i.e.

$$
\begin{gathered}
\operatorname{Pr}_{\mathbf{x}}\{f(\mathbf{x}) \leq 0\} \geq 1-p_{\text {out }} \\
\Longleftarrow\left\{\begin{array}{c}
\operatorname{tr}(\mathbf{A})+\sqrt{-2 \ln p_{\text {out }}} t_{1}-t_{2} \ln p_{\text {out }}+c \leq 0 \\
\left\|\left[\begin{array}{c}
\operatorname{vec}(\mathbf{A}) \\
\sqrt{2} \mathbf{u}
\end{array}\right]\right\|_{2} \leq t_{1} \\
t_{2} \mathbf{I}_{n}-\mathbf{A} \succeq \mathbf{0}, t_{2} \geq 0
\end{array},\right.
\end{gathered}
$$

where $t_{1}$ and $t_{2}$ are a pair of slack variables.

Firstly, by invoking Lemma 3, the wiretap rate $R_{E}$ of the EVE can be rewritten as

$$
\begin{aligned}
-R_{E}=\frac{(1-\tau)}{\ln 2} \max _{\mathbf{S} \succeq \mathbf{0}}( & -\operatorname{tr}\left[\mathbf{S}\left(\mathbf{I}_{N_{E}}+\mathbf{R}_{E}^{\frac{1}{2}} \mathbf{H}_{E} \mathbf{P}_{F} \mathbf{H}_{E}^{H} \mathbf{R}_{E}^{\frac{1}{2}}\right)\right] \\
& \left.+\ln \operatorname{det}(\mathbf{S})+N_{E}\right) .
\end{aligned}
$$

We further substitute (20) into problem (18) to obtain the reformulated constraint CR6 as

$$
\operatorname{Pr}_{\Delta \mathbf{H}_{E}}\left\{\operatorname{tr}\left(\mathbf{S R}_{E}^{\frac{1}{2}} \mathbf{H}_{E} \mathbf{P}_{F} \mathbf{H}_{E}^{H} \mathbf{R}_{E}^{\frac{1}{2}}\right)-L \leq 0\right\} \geq 1-p_{\text {out }},
$$

where $L=\ln \left(1+C_{1} \mathbf{h}_{R}^{H} \mathbf{P}_{F} \mathbf{h}_{R}\right)-\operatorname{tr}(\mathbf{S})+\ln \operatorname{det} \mathbf{S}+N_{E}-$ $\frac{\ln 2 R_{S}}{1-\tau}$. Since $\Delta \mathbf{h}_{E} \sim \mathcal{C N}\left(\mathbf{0}, \mathbf{C}_{E}\right)$, we can re-express $\Delta \mathbf{h}_{E}$ as $\Delta \mathbf{h}_{E}=\mathbf{C}_{E}^{\frac{1}{2}} \mathbf{x}_{e}$ with $\mathbf{x}_{e} \sim \mathcal{C N}\left(\mathbf{0}, \mathbf{I}_{N_{F} N_{E}}\right)$. Furthermore, through the vectorization of (21), we have 


$$
\begin{aligned}
\underset{\tilde{t}_{1}, \tilde{t}_{2}, \tau, \widetilde{\mathbf{W}}_{P} \succeq \mathbf{0}, \widetilde{\mathbf{P}}_{F} \succeq \mathbf{0}}{\max } R_{S} \\
\text { s.t. } \quad \widetilde{\mathrm{CR} 1} \sim \widetilde{\mathrm{CR} 3}, \widetilde{\mathrm{CR} 6}:\left\{\begin{array}{l}
\widetilde{\mathrm{CR} 6 a}: \operatorname{tr}\left(\widehat{\mathbf{H}}_{C E} \widetilde{\mathbf{P}}_{S}\right)+\sqrt{-2 \ln p_{\text {out }}} \tilde{t}_{1}-\tilde{t}_{2} \ln p_{\text {out }}+(1-\tau) \operatorname{tr}(\mathbf{S}) \\
-(1-\tau) \ln \operatorname{det} \mathbf{S}-(1-\tau) N_{E}+\ln 2 R_{S} \leq(1-\tau) \ln \left(1+\frac{C_{1} \mathbf{h}_{R}^{H} \widetilde{\mathbf{P}}_{F} \mathbf{h}_{R}}{1-\tau}\right) \\
\widetilde{\mathrm{CR} 6} b:\left\|\left[\begin{array}{c}
\operatorname{vec}\left(\mathbf{C}_{E}^{\frac{1}{2}} \widetilde{\mathbf{P}}_{S} \mathbf{C}_{E}^{\frac{1}{2}}\right) \\
\sqrt{2} \mathbf{C}_{E}^{\frac{1}{2}} \widetilde{\mathbf{P}}_{S} \hat{\mathbf{h}}_{E}
\end{array}\right]\right\|_{2} \leq \tilde{t}_{1} \\
\widetilde{\mathrm{CR} 6} c: \tilde{t}_{2} \mathbf{I}_{N_{F} N_{E}}-\mathbf{C}_{E}^{\frac{1}{2}} \widetilde{\mathbf{P}}_{S} \mathbf{C}_{E}^{\frac{1}{2}} \succeq \mathbf{0}, \tilde{t}_{2} \geq 0,
\end{array},\right.
\end{aligned}
$$

$$
\begin{gathered}
\operatorname{Pr}_{\Delta \mathbf{x}_{e}}\left\{\Delta \mathbf{x}_{e}^{H} \mathbf{C}_{E}^{\frac{1}{2}} \mathbf{P}_{S} \mathbf{C}_{E}^{\frac{1}{2}} \Delta \mathbf{x}_{e}+2 \operatorname{Re}\left\{\Delta \mathbf{x}_{e}^{H} \mathbf{C}_{E}^{\frac{1}{2}} \mathbf{P}_{S} \hat{\mathbf{h}}_{E}\right\}\right. \\
\left.+\hat{\mathbf{h}}_{E}^{H} \mathbf{P}_{S} \hat{\mathbf{h}}_{E}-L \leq 0\right\} \geq 1-p_{\text {out }}
\end{gathered}
$$

where $\mathbf{P}_{S}=\left(\mathbf{R}_{E}^{\frac{1}{2}} \mathbf{S} \mathbf{R}_{E}^{\frac{1}{2}}\right)^{T} \otimes \mathbf{P}_{F}$. To make the probabilistic constraint (22) tractable, we adopt a popular conservative approximation, the so-called BTI in Lemma 4, for transforming it into a series of tractable convex constraints. Then the outageconstrained SRM problem (18) is reformulated as

$$
\begin{aligned}
& \max _{t_{1}, t_{2}, \tau, \mathbf{W}_{P} \succeq \mathbf{0}, \mathbf{P}_{F} \succeq \mathbf{0}, \mathbf{S} \succeq \mathbf{0}} R_{S} \\
& \text { s.t. CR1 CR3, } \\
& \text { CR6: }\left\{\begin{array}{l}
\operatorname{tr}\left(\widehat{\mathbf{H}}_{C E} \mathbf{P}_{S}\right)+\sqrt{-2 \ln p_{\text {out }}} t_{1}-t_{2} \ln p_{\text {out }}-\ln \operatorname{det} \mathbf{S} \\
-\ln \left(1+C_{1} \mathbf{h}_{R}^{H} \mathbf{P}_{F} \mathbf{h}_{R}\right)+\operatorname{tr}(\mathbf{S})-N_{E}+\frac{\ln 2 R_{S}}{1-\tau} \leq 0 \\
\left\|\left[\begin{array}{c}
\operatorname{vec}\left(\mathbf{C}_{E}^{\frac{1}{2}} \mathbf{P}_{S} \mathbf{C}_{E}^{\frac{1}{2}}\right) \\
\sqrt{2} \mathbf{C}_{E}^{\frac{1}{2}} \mathbf{P}_{S} \hat{\mathbf{h}}_{E}
\end{array}\right]\right\|_{2} \leq t_{1} \\
\| t_{2} \mathbf{I}_{N_{F} N_{E}}-\mathbf{C}_{E}^{\frac{1}{2}} \mathbf{P}_{S} \mathbf{C}_{E}^{\frac{1}{2}} \succeq \mathbf{0}, t_{2} \geq 0
\end{array}\right.
\end{aligned}
$$

where $\widehat{\mathbf{H}}_{C E}=\hat{\mathbf{h}}_{E} \hat{\mathbf{h}}_{E}^{H}+\mathbf{C}_{E}$. Although problem (23) is still not jointly concave w.r.t $\left\{\tau, \mathbf{W}_{P}, \mathbf{P}_{F}, \mathbf{S}\right\}$, it is more tractable than the outage-constrained SRM problem (18). Specifically, by utilizing $\widetilde{\mathbf{W}}_{P}=\tau \mathbf{W}_{P}, \widetilde{\mathbf{P}}_{F}=(1-\tau) \mathbf{P}_{F}$ and $\widetilde{\mathbf{S}}=\tau \mathbf{S}$, we easily find that problem (23) is jointly concave w.r.t. $\left\{\tau, \widetilde{\mathbf{W}}_{P}, \widetilde{\mathbf{P}}_{F}\right\}$ when fixing $\mathbf{S}$, which is similar to problems (8) and (17). In turn, problem (23) is also concave w.r.t. $\mathbf{S}$ when fixing $\left\{\tau, \mathbf{W}_{P}, \mathbf{P}_{F}\right\}$. Interestingly, we also validate that problem (23) admits the optimal rank-1 solution, as shown in the following Theorem.

Theorem 5. The optimal solution $\left\{\mathbf{W}_{P}^{\star}, \mathbf{P}_{F}^{\star}\right\}$ to problem (23) $\operatorname{satisfies} \operatorname{rank}\left(\mathbf{W}_{P}^{\star}\right)=\operatorname{rank}\left(\mathbf{P}_{F}^{\star}\right)=1$, which is also a highquality solution for the computationally intractable problem (18).

Proof. Please refer to Appendix C.

Based on the above analysis, we propose an alternating optimization procedure for efficiently solving problem (23) in the sequel. The first subproblem for optimizing $\left\{\tilde{t}_{1}, \tilde{t}_{2}, \tau, \widetilde{\mathbf{W}}_{P}, \widetilde{\mathbf{P}}_{F}\right\}$ given $\mathbf{S}$ is expressed as problem (24) (shown at the top of the next page), where $\widetilde{\mathbf{P}}_{S}=$ $\left(\mathbf{R}_{E}^{\frac{1}{2}} \mathbf{S R}_{E}^{\frac{1}{2}}\right)^{T} \otimes \widetilde{\mathbf{P}}_{F}$. Due to the joint concavity of problem (24), we can optimally recover a high-quality suboptimal solution

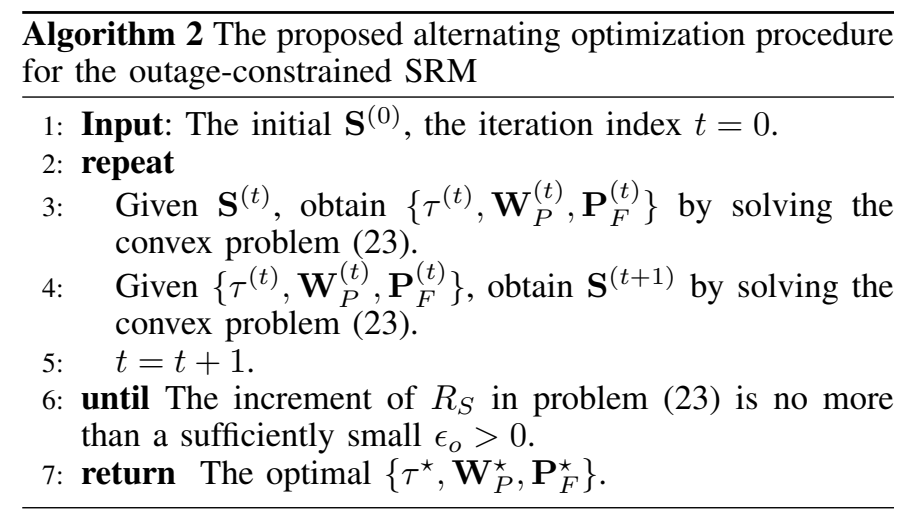

$\left\{\tau, \mathbf{W}_{P}, \mathbf{P}_{F}\right\}$ to problem (18) from the obtained optimal rank1 solution of problem (24). Then the second subproblem for optimizing $\mathbf{S}$ given $\left\{\tau, \mathbf{W}_{P}, \mathbf{P}_{F}\right\}$ is formulated as

$$
\max _{\tilde{t}_{1}, \tilde{t}_{2}, \mathbf{S} \succeq \mathbf{0}} R_{S}, \quad \text { s.t. } \mathrm{CR} 1 \sim \mathrm{CR} 3, \widetilde{\mathrm{CR} 6} .
$$

Notice that problem (25) is also jointly concave w.r.t. $\left\{\tilde{t}_{1}, \tilde{t}_{2}, \mathbf{S} \succeq \mathbf{0}\right\}$, and thus can be globally addressed by the standard convex optimization technique. Overall, the outageconstrained SRM problem (18) can be effectively solved by the proposed alternating optimization procedure summarized in Algorithm 2.

\section{Secure Communications under Completely UNKNOWN FBS-EVE CSI}

In the previous two Sections, both full and partial FBSEVE CSI are considered for jointly optimizing the PB energy covariance matrix, the FBS information covariance matrix and the time splitting factor. Nevertheless, in practice, the EVE may be passive and the FBS is unaware of the existence of the EVE. In this context, we accordingly propose AN aided scheme for secrecy wirelessly powered HetNets. With this scheme, the FBS simultaneously transmits the confidential signal $\mathbf{s}_{F}$ and $\mathrm{AN} \mathbf{z}_{F}$ to the FU, i.e., $\mathbf{x}_{F}=\mathbf{s}_{F}+\mathbf{z}_{F}$, for sufficiently interfering the EVE without affecting the FU. Hence, the generated AN should be in the null-space of the FBS-FU channel, i.e., $\mathbf{h}_{R} \mathbf{z}_{F}=\mathbf{0}$. We then express the AN as $\mathbf{z}_{F}=\mathbf{H}_{R}^{\perp} \mathbf{n}_{F}$, where $\mathbf{H}_{R}^{\perp} \in \mathbb{C}^{N_{F} \times\left(N_{F}-1\right)}$ denotes the orthogonal complement space of $\mathbf{h}_{R}$ and $\mathbf{n}_{F} \sim \mathcal{C N}\left(\mathbf{0}, \boldsymbol{\Sigma}_{U}\right)$ is a novel $\left(N_{F}-1\right)$-dimensional AN vector with the positive semidefinite covariance matrix $\boldsymbol{\Sigma}_{U} \succeq \mathbf{0}$. Since the FBS-EVE CSI is unavailable at the FBS, the direct secrecy rate optimization of the considered wirelessly powered HetNet is infeasible. As an alternative, we consider maximizing the average $\mathrm{AN}$ power 


$$
\begin{aligned}
\max _{\tau, \widetilde{\mathbf{W}}_{P} \succeq \mathbf{0}, \widetilde{\mathbf{P}}_{F} \succeq \mathbf{0}, \widetilde{\boldsymbol{\Sigma}}_{U} \succeq \mathbf{0}} \operatorname{tr}\left(\widetilde{\boldsymbol{\Sigma}}_{U}\right) \\
\text { s.t. } \widetilde{\mathrm{CR} 1}, \widetilde{\mathrm{CR} 3}, \widetilde{\mathrm{AR} 2}:(1-\tau) \log _{2}\left(1+\frac{C_{1} \mathbf{h}_{R}^{H} \widetilde{\mathbf{P}}_{F} \mathbf{h}_{R}}{1-\tau}\right) \geq R_{t h} \\
\widetilde{\mathrm{AR} 3}: \operatorname{tr}\left(\widetilde{\mathbf{P}}_{F}+\widetilde{\mathbf{\Sigma}}_{U}\right) \leq \xi \operatorname{tr}\left(\mathbf{H}_{F} \widetilde{\mathbf{W}} \widetilde{H}_{F}^{H}\right)+\xi \tau \frac{P_{M}}{N_{M}} \operatorname{tr}\left(\mathbf{G}_{F} \mathbf{G}_{F}^{H}\right) .
\end{aligned}
$$

$(1-\tau) \operatorname{tr}\left(\boldsymbol{\Sigma}_{U}\right)$ at the EVE subject to the minimum legitimate rate at the $\mathrm{FU}$, so as to impose as much interference as possible on the potential EVE for reducing its wiretapping capability. The AN aided secrecy problem is ultimately formulated as

$$
\begin{aligned}
& \max _{\tau, \mathbf{W}_{P} \succeq \mathbf{0}, \mathbf{P}_{F} \succeq \mathbf{0}, \boldsymbol{\Sigma}_{U} \succeq \mathbf{0}} \quad(1-\tau) \operatorname{tr}\left(\boldsymbol{\Sigma}_{U}\right) \\
& \text { s.t. CR1, CR3, } \\
& \text { AR2 : }(1-\tau) \log _{2}\left(1+\frac{\mathbf{h}_{R}^{H} \mathbf{P}_{F} \mathbf{h}_{R}}{\sigma_{n}^{2}+\frac{P_{M}}{N_{M}}\left\|\mathbf{g}_{R}\right\|^{2}}\right) \geq R_{t h} \\
& \text { AR3 : }(1-\tau) \operatorname{tr}\left(\mathbf{P}_{F}+\boldsymbol{\Sigma}_{U}\right) \\
& \leq \tau \xi\left[\operatorname{tr}\left(\mathbf{H}_{F} \mathbf{W}_{P} \mathbf{H}_{F}^{H}\right)+\frac{P_{M}}{N_{M}} \operatorname{tr}\left(\mathbf{G}_{F} \mathbf{G}_{F}^{H}\right)\right]
\end{aligned}
$$

Naturally, problem (26) with coupled variables is not jointly concave w.r.t $\left\{\tau, \mathbf{W}_{P}, \mathbf{P}_{F}, \boldsymbol{\Sigma}_{U}\right\}$. Based on the definitions $\widetilde{\mathbf{W}}_{P}=\tau \mathbf{W}_{P}, \widetilde{\mathbf{P}}_{F}=(1-\tau) \mathbf{P}_{F}$ and $\widetilde{\boldsymbol{\Sigma}}_{U}=(1-\tau) \boldsymbol{\Sigma}_{U}$, problem (26) can be rewritten as problem (27), as shown at the top of the next page. Similar to problems (8) and (17), problem (27) composed of a linear objective function and concave constraints is also jointly concave w.r.t $\left\{\tau, \widetilde{\mathbf{W}}_{P}, \widetilde{\mathbf{P}}_{F}, \widetilde{\boldsymbol{\Sigma}}_{U}\right\}$, and thus can be optimally solved. Moreover, the optimal rank-1 solution $\left\{\widetilde{\mathbf{W}}_{P}^{\star}, \widetilde{\mathbf{P}}_{F}^{\star}, \widetilde{\boldsymbol{\Sigma}}_{U}^{\star}\right\}$ to problem (27) is revealed in the following Theorem.

Theorem 6. There always exists an optimal solution $\left\{\mathbf{W}_{P}^{\star}, \mathbf{P}_{F}^{\star}, \boldsymbol{\Sigma}_{U}^{\star}\right\}$ with $\operatorname{rank}\left(\mathbf{W}_{P}^{\star}\right)=\operatorname{rank}\left(\mathbf{P}_{F}^{\star}\right)=\operatorname{rank}\left(\boldsymbol{\Sigma}_{U}^{\star}\right)=$ 1 for the AN aided secrecy problem (26).

Proof. Please refer to Appendix D.

Theorem 7. For all the above cases of FBS-EVE CSI, if the cross-interference constraint CR3 is inactive, a closed-form expression for the $P B$ energy covariance matrix can be derived as $\mathbf{W}_{P}^{\star}=P_{P} \mathbf{w}_{P} \mathbf{w}_{P}^{H}$ with $\mathbf{w}_{P}=\nu_{\max }\left(\mathbf{H}_{F}^{H} \mathbf{H}_{F}\right)$.

Proof. Please refer to Appendix E.

\section{COMPlexity ANALysis AND EXTENSIONS OF OUR WORK}

\section{A. Convergence and Complexity Analysis}

As mentioned above, the proposed convexity-based line search is applicable to both the perfect SRM problem (5) and worst-case SRM problem (12). Moreover, benefited from the quasi-convexity and convexity of the two problems w.r.t $\eta$ and $\left\{\mathbf{W}_{P}, \mathbf{P}_{F}, \boldsymbol{\Sigma}_{U}\right\}$ given any $\eta$, respectively, we easily infer that the proposed convexity-based line search converges to the globally optimal solution to each problem. Next, for the outage-constrained SRM problem (24), an alternating optimization procedure is proposed and guaranteed to converge to a locally optimal solution, since the achievable secrecy rate is non-decreasing within the whole iterative process and the feasible region of problem (23) is closed and finite. Finally, the proposed AN aided secrecy optimization problem (26) can also be globally solved, since it can be equivalently transformed into a jointly convex counterpart.

Next, we firstly discuss the computational complexity of Algorithm 1 and Algorithm 2 for the perfect SRM problem (5) (worst-case SRM problem (12)) and the outage-constrained SRM problem (24), respectively, where both the low-order and the constant terms are omitted. Specifically, for problem (5)/(12), the complexity of Algorithm 1 comes mainly from the Golden section search for finding the optimal $\eta^{\star}$ and the semidefinite cone constraints in problem $(8) /(17)$. It is known that the number of iterations required by Golden section search is $\log \left(\frac{\eta_{\max }-\eta_{\min }}{\epsilon_{a}}\right)$, where $\epsilon_{a}$ denotes the search accuracy. Additionally, by referring to [34], the computational complexity order of solving a standard SDP problem is $\mathcal{O}\left(M_{s d p} N_{s d p}^{3.5}+M_{s d p}^{2} N_{s d p}^{2.5}+M_{s d p}^{3} N_{s d p}^{0.5}\right) \log \left(\frac{1}{\epsilon_{s}}\right)$, where $M_{s d p}$ and $N_{s d p}$ denote the number of semidefinite cone constraints and the dimension of the semidefinite cone, respectively, while $\epsilon_{s}$ is the desired accuracy. Therefore, problems (8) and (17) can be solved in each iteration at the complexity order of $\mathcal{O}\left(N_{F}^{3.5}+N_{P}^{3.5}\right) \log \left(\frac{1}{\epsilon_{s}}\right)$ and $\mathcal{O}\left(\left(N_{E} N_{F}\right)^{3.5}+N_{P}^{3.5}\right) \log \left(\frac{1}{\epsilon_{s}}\right)$, respectively. Correspondingly, the total complexity order of Algorithm 1 aiming for solving problems (5) and (12) is given by $\log \left(\frac{\eta_{\max }-\eta_{\min }}{\epsilon_{a}}\right) \mathcal{O}\left(\left(N_{F}^{3.5}+N_{P}^{3.5}\right) \log \left(\frac{1}{\epsilon_{s}}\right)\right)$ and $\log \left(\frac{\eta_{\max }-\eta_{\min }}{\epsilon_{a}}\right) \mathcal{O}\left(\left(\left(N_{E} N_{F}\right)^{3.5}+N_{P}^{3.5}\right) \log \left(\frac{1}{\epsilon_{s}}\right)\right)$, respectively. Furthermore, the complexity of Algorithm 2 aiming at iteratively solving a pair of convex subproblems (23), each of which has a second-order cone constraint of dimension $\left(N_{E} N_{F}\right)^{2}+N_{E} N_{F}+1$, is given by $\mathcal{O}\left(I_{A}\left(N_{E} N_{F}\right)^{4}\left(N_{E}^{2}+\right.\right.$ $\left.\left.N_{F}^{2}\right)\right) \log \left(\frac{1}{\epsilon_{s}}\right)$, where $I_{A}$ denotes the number of iterations [34]. Similarly, the AN aided secrecy problem (27) belongs to the SDP with the complexity order of $\mathcal{O}\left(N_{F}^{3.5}+N_{P}^{3.5}\right) \log \left(\frac{1}{\epsilon_{s}}\right)$.

\section{B. Extensions to the case of multiple communication nodes}

Firstly, for the case of multiple FBSs serving different FUs, if the eavesdropper only aims for intercepting the signal from a specific FBS, all our proposed secrecy beamforming designs are directly applicable by simply regarding the signals from other undesired FBSs as interference. However, if the information leakage occurs between all FBSs and the eavesdropper, the extension of our work will become quite challenging, because the transmit beamforming design of each FBS has to consider maximizing the secrecy rate and minimizing the resultant interference to other unintended FUs simultaneously. In this context, both perfect and robust secrecy beamforming designs are more challenging to address due to the existence 
of strongly coupled variables. Next, considering that energy harvesting only occurs during the initial $\tau T$ subslot, which is independent of the secure transmission of the FBS in the second subslot, it may be readily inferred that all proposed secrecy beamforming designs can be directly applied to the case of multiple PBs. Refer to [12], all the above proposed secrecy beamforming designs can be readily extended to the multiple EVEs case, in which the system secrecy rate becomes the minimum secrecy rate in the presence of multiple eavesdroppers. In this context, the rank-1 optimality of both the PB energy and FBS information secrecy beamformings still holds by following similar proofs to that in our work. Based on the above analysis, we further infer that for the coexistence of multiple PBs, multiple FBSs and multiple eavesdroppers, the intractability of secrecy beamforming optimization is mainly due to the inter-user interference among multiple FUs associated with different FBSs.

\section{Simulation Results and Discussions}

In this section, numerical simulation results are provided for quantifying the secrecy rate performance of the wirelessly powered HetNet. Unless otherwise stated, in the following simulations the MBS, the $\mathrm{PB}$, the FBS and the EVE are equipped with $N_{M}=2, N_{P}=4, N_{F}=4$ and $N_{E}=3$ antennas, respectively. Furthermore, a single-antenna MU and a single-antenna FU are considered. The locations of the MBS, the MU and the PB are $(0,0) \mathrm{m},(150,0) \mathrm{m}$ and $(100,100) \mathrm{m}$, respectively. Since the FBS mainly harvests wireless energy from the PB, the FBS location is near the PB and thus set as $(105,105) \mathrm{m}$. Moreover, the FU and the EVE are located at $(105,200) \mathrm{m}$ and $(155,105) \mathrm{m}$, respectively. For all wireless channels, the path loss model is determined as $\mathrm{L}_{0}=10^{-3} d^{-\alpha}$, where $L_{0}$ denotes the signal power loss at a distance $d$ between two arbitrary terminals and $\alpha=2$ is the path loss exponent [18]. Notice that in this model, a $30 \mathrm{~dB}$ average signal power loss at $d=1 \mathrm{~m}$ is implied. Moreover, the smallscale fading of all channel coefficients is modeled as an i.i.d. Rayleigh distribution with zero mean and unit variance. The Gaussian noise variance is set as $\sigma_{n}^{2}=-110 \mathrm{dBm}$. The maximum transmit power of the MBS and $\mathrm{PB}$ are defined as $P_{M}=10 \mathrm{dBm}$ and $P_{P}=20 \mathrm{dBm}$, respectively. The FBS energy harvesting efficiency is $\xi=0.8$. Initially, we fix the interference threshold to be $I_{t h}=-65 \mathrm{dBm}$. For the worstcase SRM (WSRM), the bound of FBS-EVE CSI error is set as $\xi_{f}=0.2$. By contrast, for the outage-constrained SRM (OSRM), the covariance matrix of FBS-EVE CSI error $\mathbf{C}_{E}$ and the outage probability $p_{\text {out }}$ are defined as $\mathbf{C}_{E}=\zeta^{2} \mathbf{I}_{N_{E}}$ with $\zeta=0.05$ and $p_{\text {out }}=0.1$, respectively. Finally, for the proposed AN scheme, an legitimate rate threshold at the FU is chosen as $R_{t h}=\frac{R_{\max }}{2}$, where $R_{\max }$ denotes the maximum achievable rate of the wirelessly powered HetNet without the EVE and can be optimally derived according to [13]. Note that all simulation points are plotted by averaging over 200 Monte-Carlo experiments.

Additionally, we consider four benchmarks for the proposed secrecy beamforming designs under three different levels of FBS-EVE CSI. Specifically, for both the perfect and unknown
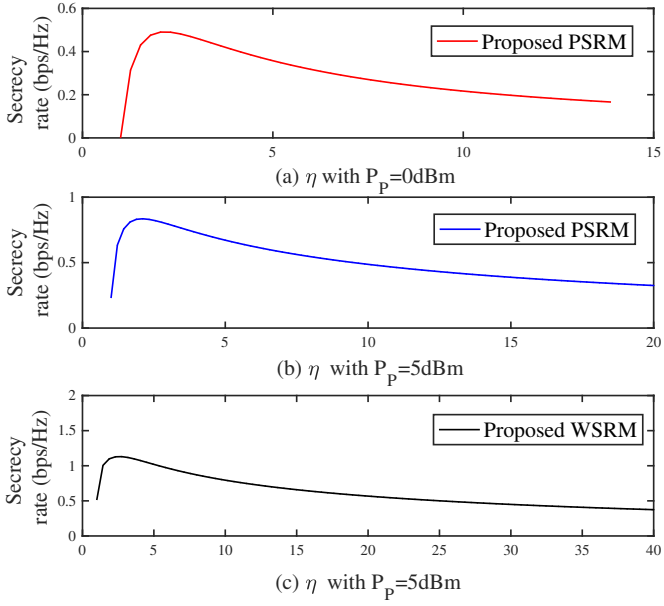

Fig. 2. Achievable secrecy rates for the proposed PSRM and WSRM versus the parameter $\eta$ to validate Theorem 2 .

FBS-EVE CSI cases, the equal power allocation schemes are adopted at the FBS ( also referred to as FBS-EPA) with the fixed and optimized time allocation, i.e. $\tau=\frac{1}{2}$ and $\tau=\tau^{\star}$, respectively. Moreover, both the two benchmarks assume $\mathbf{W}_{P}=\frac{P_{P}}{N_{P}} \mathbf{I}_{N_{P}}$. While for the imperfect FBS-EVE CSI, the nonrobust SRM scheme is adopted as a benchmark for the proposed WSRM, where the achievable secrecy rate is calculated by substituting the optimal solution $\left\{\tau, \mathbf{W}_{P}, \mathbf{P}_{F}\right\}$ derived when $\xi=0$ into the worst-case FBSEVE channel. Furthermore, by referring to [28], the worstcase SRM with the error bound $\xi=\sqrt{\frac{\zeta^{2}}{2} F_{\chi_{2 N_{F} N_{E}}^{2}}^{-1}\left(1-p_{\text {out }}\right)}$ is actually a conservative approximation for the proposed OSRM, where $F_{\chi_{2 N_{F} N_{E}}^{2}}^{-1}(\cdot)$ denotes the inverse cumulative density function (CDF) of a Chisquare random variable with degrees of freedom $2 N_{F} N_{E}$. Therefore, we also consider it to be a benchmark for the proposed OSRM. Notice that if the predefined interference level $I_{t h}$ is not realized by the adopted benchmarks, the corresponding achievable secrecy rate is set to zero.

\section{A. The Proposed Perfect and Worst-Case SRMs}

In this subsection, we evaluate the achievable secrecy rates of the proposed PSRM and WSRM in Section III and Section IV-A corresponding to problem (8) and problem (17), respectively. Firstly, to validate Theorem 2, the achievable secrecy rates of the above two schemes over a feasible range of $\eta$ are shown in Fig. 2, where different PB transmit power $P_{P}=0,5 \mathrm{dBm}$ are considered. It is clearly observed from Fig. 2 that both the PSRM problem (8) and WSRM problem (17) are unimodal (quasi-concave) functions of $\eta$, and thus the globally optimal $\eta$ can be determined via the GSS.

Fig. 3 depicts the achievable secrecy rates of all schemes versus the $\mathrm{PB}$ transmit power $P_{P}$ with different numbers of EVE's antennas $N_{E}$. From Fig. 3 (a) with $N_{E}=3$, we readily find that the achievable secrecy rates of all schemes increase with $P_{P}$. The proposed PSRM realizes the highest secrecy rate among all schemes. While the proposed WSRM 


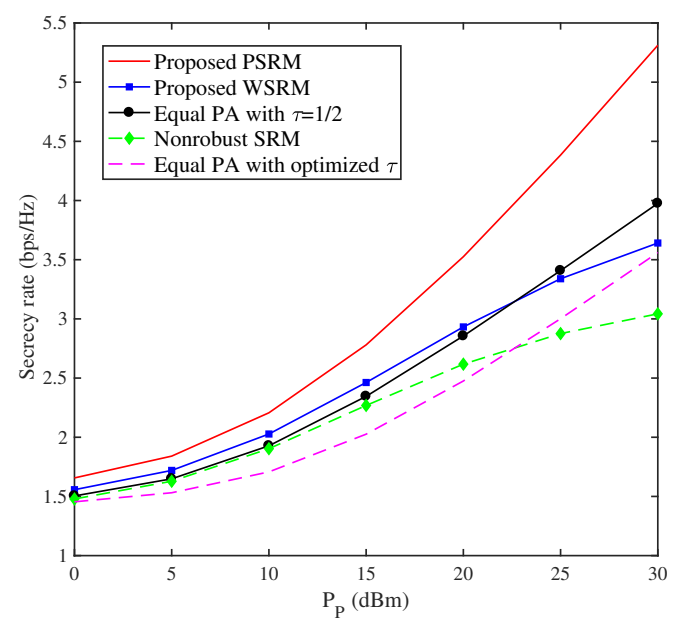

(a) $N_{E}=3$

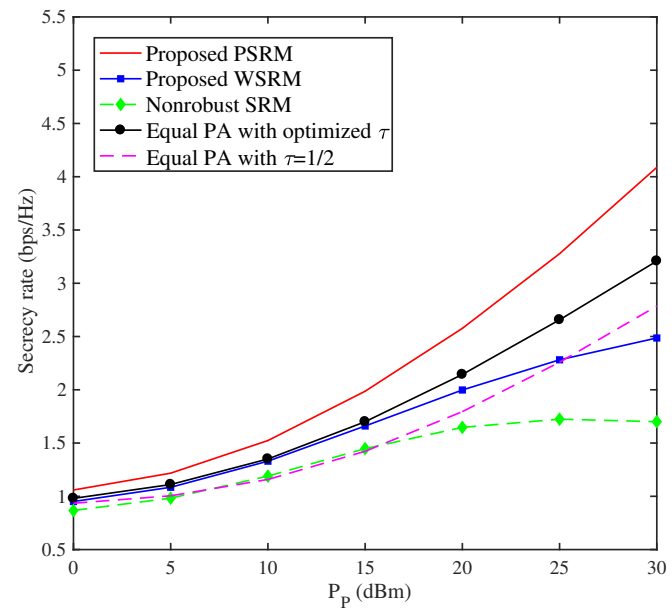

(b) $N_{E}=4$

Fig. 3. Achievable secrecy rates for different schemes versus the PB transmit power $P_{P}$ with (a) $N_{E}=3$ and (b) $N_{E}=4$.

considering the influence of practical CSI error on the secrecy beamforming design naturally performs better than the nonrobust counterpart. By comparing the two adopted FBSEPA benchmarks, we clearly see that time allocation plays an important role in improving secrecy rate performance of the wirelessly powered HetNet. Furthermore, in Fig. 3(b) with $N_{E}=4$, the same comparisons among all schemes as in Fig. 3 (a) can be observed. Moreover, each scheme achieves a lower secrecy rate than its counterpart in Fig. 3 (a) due to the increased EVE's wiretap capability.

Next, Fig. 4 (a) investigates the achievable secrecy rates of all schemes versus the interference level $I_{t h}$. We clearly find from Fig. 4(a) that the secrecy rate value for each scheme firstly increases with $I_{t h}$ until $I_{t h}$ reaches a certain threshold $I_{t h}^{m i}$, then becomes saturated. Here, $I_{t h}^{m i}$ represents the optimal solution of the PSRM problem (8) or the WSRM problem (17) with the interference constraint neglected. This is because that when a small $0<I_{t h} \leq I_{t h}^{m i}$ is adopted, it is easily inferred that the achievable secrecy rates of all schemes are dominated by the cross-interference constraint. In this context, since the feasible regions of both problems (8) and (17) expand

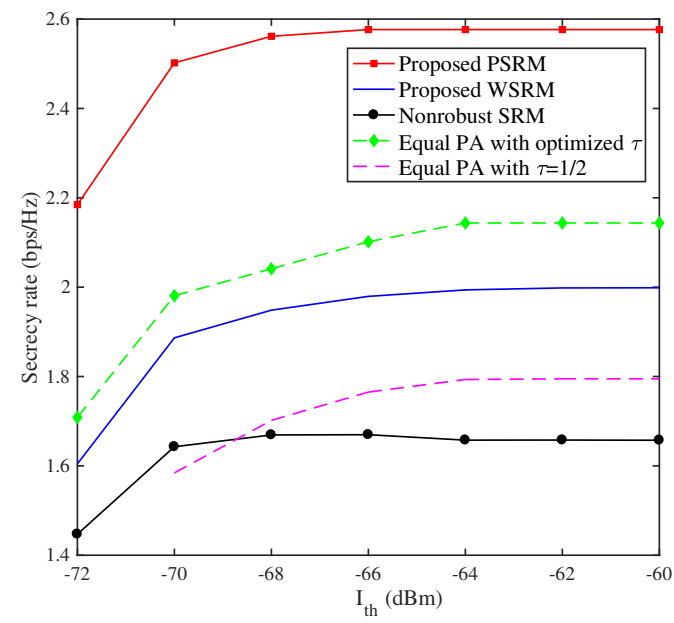

(a) $N_{E}=4, P_{P}=20 \mathrm{dBm}$

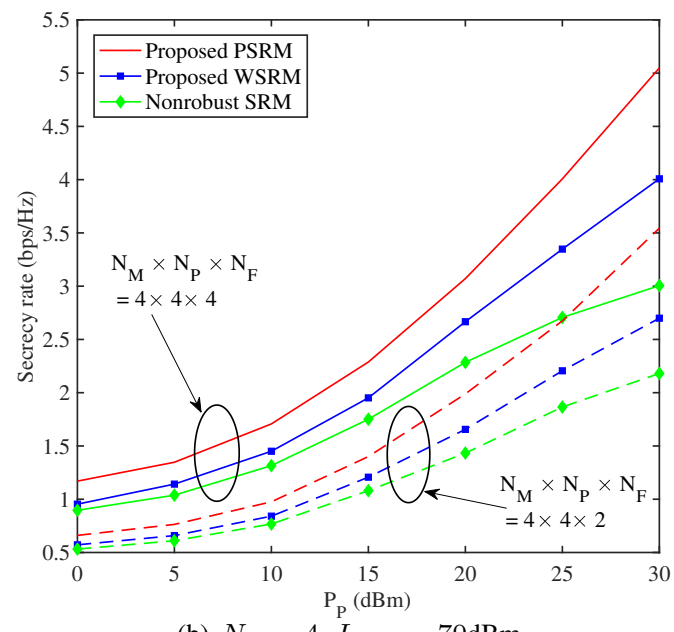

(b) $N_{E}=4, I_{t h}=-70 \mathrm{dBm}$

Fig. 4. Achievable secrecy rates for different schemes versus (a) the interference level $I_{t h}$; (b) the PB transmit power $P_{P}$.

with the increasing $I_{t h}$, the corresponding achievable secrecy rates also increase. However, when $I_{t h}$ becomes sufficiently large, i.e $I_{t h}>I_{t h}^{m i}$, the cross-interference constraint actually becomes inactive and thus has no effect on achievable secrecy rates. That is to say, the achievable secrecy rates become saturated regardless of the increasing $I_{t h}$ when $I_{t h}>I_{t h}^{m i}$. In particular, the saturated secrecy rate value can be derived from problem $(8)((17))$ by neglecting the cross-interference constraint. Additionally, the proposed PSRM still has the best secrecy rate performance among all schemes and the proposed WSRM provides much higher secrecy rate than the nonrobust SRM. Fig. 4 (b) further illustrates the achievable secrecy rates of the proposed PSRM and WSRM versus the PB transmit power $P_{P}$, where $I_{t h}=-70 \mathrm{dBm}$ and $N_{E}=4$. For all studied schemes, we firstly find that the achievable secrecy rate in the case of $N_{M} \times N_{P} \times N_{F}=2 \times 4 \times 4$ shown in Fig. 3(b) is lower than that of $N_{M} \times N_{P} \times N_{F}=4 \times 4 \times 4$, which may be attributed to both the more harvested energy at the FBS and stronger interference at the EVE brought by the increasing number of MBS antennas $N_{M}$. Nevertheless, the worst secrecy performance is observed in the case of $N_{M} \times N_{P} \times N_{F}=4 \times 4 \times 2$, 


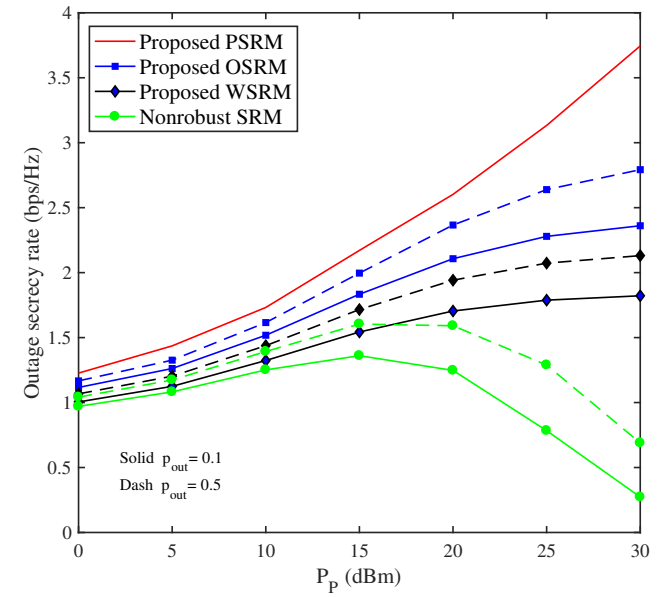

Fig. 5. Achievable secrecy rates for different schemes versus the PB transmit power $P_{P}$ under different outage probabilities.

since a reduced number of FBS antennas $N_{F}$ generally leads to less harvested energy at the FBS. Furthermore, we observe from Fig. 4(b) that as a benefit of high-SNR transmission, the performance gap between the proposed WSRM and the nonrobust SRM increases with $P_{P}$ in both two cases.

\section{B. The Proposed Outage-Constrained SRM}

In this subsection, we investigate the achievable secrecy rates of the proposed OSRM, i.e. problem (18) in Section IV. B. As mentioned before, the proposed WSRM with FBSEVE's CSI error bound $\xi_{f}=\sqrt{\frac{\zeta^{2}}{2} F_{\chi_{2 N_{F} N_{E}}^{2}}^{-1}\left(1-p_{\text {out }}\right)}$ can be regarded as another conservation reformulation for the intractable outage probability constraint, so we also adopt it as a benchmark for fair comparisons hereafter. Fig. 5 firstly shows secrecy rate performance for all schemes versus $\mathrm{PB}$ transmit power $P_{P}$ under different outage probabilities $p_{\text {out }}=0.1,0.5$. We firstly observe from Fig. 5 that for both considered $p_{\text {out }}$, the achievable secrecy rates of the proposed PSRM, WSRM and OSRM all increase with $P_{P}$. Meanwhile, the proposed OSRM has a better secrecy rate performance than the proposed WSRM, while the nonrobust SRM performs worst due to the ignorance of CSI error. Furthermore, we find that for each scheme the higher secrecy rate is realized with the outage probability $p_{\text {out }}=0.5$. This is because that when the outage tolerance is relaxed, i.e. from $p_{\text {out }}=0.1$ to $p_{\text {out }}=0.5$, the corresponding secrecy outage rate threshold also becomes higher.

Next, Fig. 6(a) illustrates secrecy rate performance of all schemes versus the CSI error variance $\zeta$ with different interference levels $I_{t h}=-65,-70 \mathrm{dBm}$. On the one hand, for each $I_{t h}$, we observe that the achievable secrecy rates of all schemes decrease with the increasing $\zeta$. The proposed OSRM still has better secrecy rate performance than the proposed WSRM. While the nonrobust SRM performs the worst. Moreover, the performance gaps between the proposed OSRM and the other two schemes both become larger when $\zeta$ increases. On the other hand, the higher secrecy rates of all schemes are observed at the interference level $I_{t h}=-65 \mathrm{dBm}$

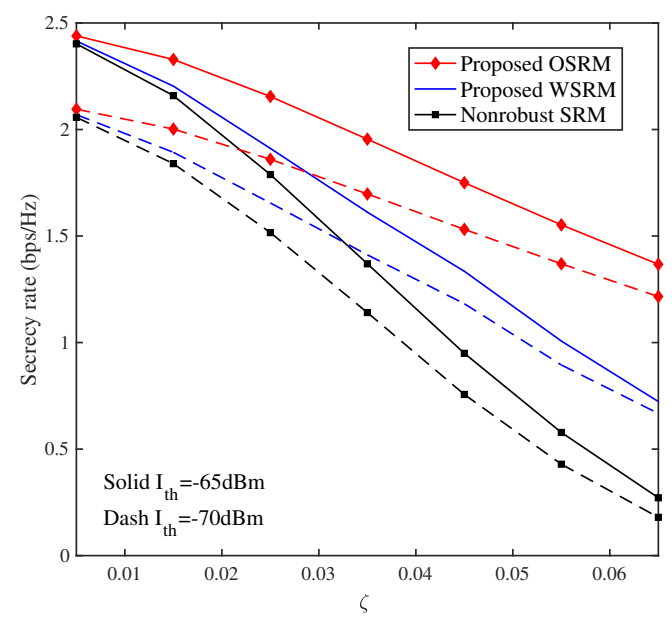

(a)

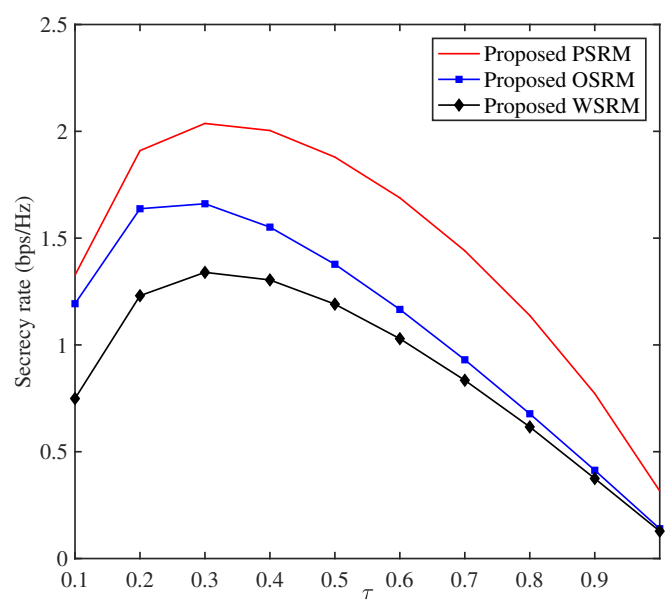

(b)

Fig. 6. Achievable secrecy rates for different schemes versus (a) the CSI error variance $\zeta$; (b) the time allocation $\tau$.

due to the larger feasible region of our secrecy beamforming design. Based on $I_{t h}=-65 \mathrm{dBm}$, Fig. 6 (b) further depicts the achievable secrecy rates of the proposed perfect and robust designs as a function of the time splitting factor $\tau$. It is clear from Fig. 6 (b) that the achievable secrecy rate is indeed concave w.r.t. $\tau$ for all studied schemes, which confirms the theoretical analysis of Section III and Section IV. As expected, the proposed PSRM performs best in terms of the achievable secrecy rate, while the proposed WSRM performs worst since it in essence represents a conservative approximation for the outage-constrained robust design.

\section{The proposed AN scheme}

In this subsection, our goal is to illustrate the optimal artificial noise power (ANP) of the proposed AN scheme in Section V. In Fig. 7 (a), the optimized ANP for different schemes versus the $\mathrm{PB}$ transmit power $P_{P}$ with different interference thresholds $I_{t h}=-70,+\infty$ is shown, where the legitimate rate threshold is set as $R_{t h}=1.5(\mathrm{bps} / \mathrm{Hz})$. Notice that for some small $P_{P}$, by which the legitimate rate threshold $R_{t h}$ is not supported, we correspondingly set 


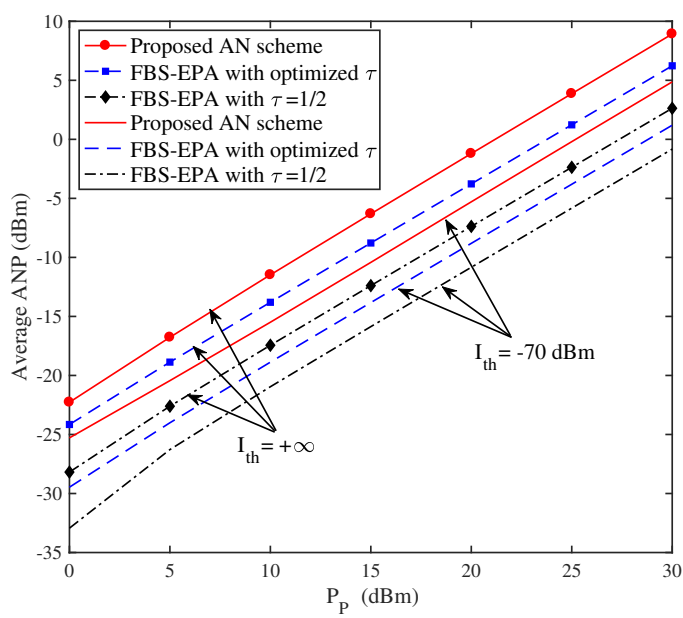

(a) $R_{t h}=1.5(\mathrm{bps} / \mathrm{Hz})$

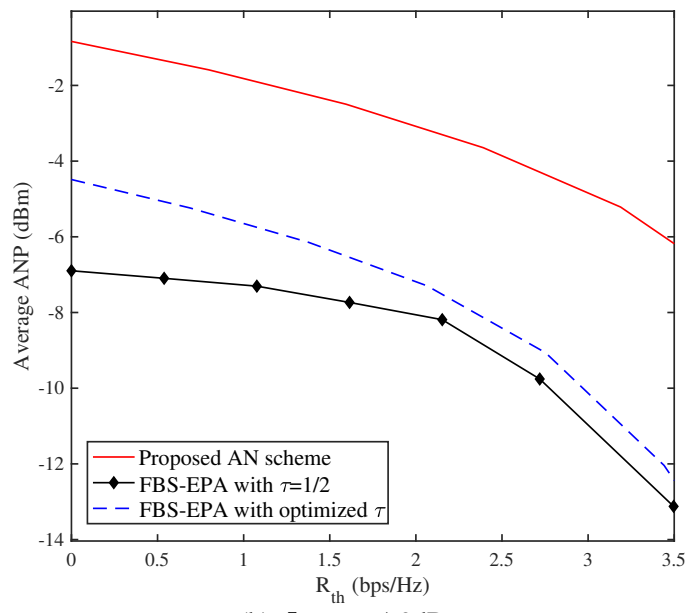

(b) $I_{t h}=-70 \mathrm{dBm}$

Fig. 7. Artificial noise power for different schemes versus (a) the PB transmit power $P_{P}$ under different interference levels $I_{t h}$; (b) the legitimate rate threshold $R_{t h}$.

the achievable secrecy rate to be zero. We firstly find from Fig. 7 (a) the achieved ANP increases with $P_{P}$ for all three schemes. Moreover, the proposed AN scheme has the highest ANP compared to the other two schemes, implying that the strongest interference is imposed on the EVE to reduce its wiretapping capability. Fig. 7 (b) shows the optimal ANP for different schemes versus the legitimate rate threshold $R_{t h}$. It is clear from Fig. 7 (b) that for each scheme, the optimized ANP decreases with the increasing $R_{t h}$ since the more portion of PB transmit power $P_{P}$ is allocated for information transfer to the FU for achieving the rate threshold $R_{t h}$. Furthermore, the proposed AN scheme still realizes the highest ANP among all schemes, which promotes secure communications of the wirelessly powered HetNet via sufficiently interfering the malicious EVE.

\section{CONCLUSIONS}

In this paper, we investigated secure communications of the wirelessly powered HetNet from different perspectives of the FBS-EVE CSI. Firstly, in the case of perfect FBSEVE CSI, the perfect and worst-case SRMs was studied by jointly optimizing the PB energy and FBS information covariance matrices as well as the time splitting factor, which can be globally addressed by the proposed convexity-based line search. Secondly, considering deterministically and statistically imperfect cases of FBS-EVE CSI, we applied Sprocedure and BTI to transform intractable CSI error related constraints into a series of tractable convex ones, respectively. Finally, for the completely unknown FBS-EVE CSI case, an AN aided secrecy beamforming design was proposed to interfere the EVE as much as possible. In particular, when the cross-interference constraint is negligible, the closed-form PB energy covariance matrix for all considered cases can be derived. More importantly, we also proved the rank-1 property of the optimal solutions for all studied SRM problems. Numerical experiments verified the excellent secrecy performance of all our proposed secrecy beamforming designs.

\section{APPENDIX A}

\section{A. The proof of Theorem 1}

Theorem 1 is proved by contradiction as follows. Firstly, we consider an interference threshold $I_{t h}^{1}$ satisfying $0 \leq I_{t h}^{1} \leq \tilde{I}_{t h}$ and denote the obtained $\tilde{R}_{S}^{\star}$ by solving problem (8) as $\tilde{R}_{S}^{\star}=$ $f_{o b j, I_{t h}^{1}}^{\star}\left(\mathcal{Q}_{1}\right)$, where $\mathcal{Q}_{1}=\left\{\widetilde{\mathbf{P}}_{F, 1}^{\star}, \widetilde{\mathbf{W}}_{P, 1}^{\star}, \tau_{1}^{\star}, \eta_{1}^{\star}\right\}$ is the optimal solution to problem (8) with $I_{t h}=I_{t h}^{1}$ and $\mathbf{h}_{M}^{H} \widetilde{\mathbf{W}}_{P, 1}^{\star} \mathbf{h}_{M}+$ $\mathbf{g}_{p}^{H} \widetilde{\mathbf{P}}_{F, 1}^{\star} \mathbf{g}_{p}<I_{t h}^{1}$ is assumed. It is then easily found that there is another interference threshold $I_{t h}^{2}$ satisfying $I_{t h}^{2}=$ $\mathbf{h}_{M}^{H} \widetilde{\mathbf{W}}_{P, 1}^{\star} \mathbf{h}_{M}+\mathbf{g}_{p}^{H} \widetilde{\mathbf{P}}_{F, 1}^{\star} \mathbf{g}_{p}<I_{t h}^{1}$, based on which the optimal solution $\mathcal{Q}_{1}$ actually becomes a feasible solution to problem (8). In other words, we have $f_{o b j, I_{t h}^{1}}^{\star}\left(\mathcal{Q}_{1}\right) \leq f_{o b j, I_{t h}^{2}}^{\star}\left(\mathcal{Q}_{2}\right)$, where $\mathcal{Q}_{2}=\left\{\widetilde{\mathbf{P}}_{F, 2}^{\star}, \widetilde{\mathbf{W}}_{P, 2}^{\star}, \tau_{2}^{\star}, \eta_{2}^{\star}\right\}$ is the optimal solution to problem (8) with $I_{t h}=I_{t h}^{2}$. On the other hand, since $I_{t h}^{2}<I_{t h}^{1}$, a smaller feasible region is observed for problem (8) with $I_{t h}=I_{t h}^{2}$, which thus yields $f_{o b j, I_{t h}^{1}}^{\star}\left(\mathcal{Q}_{1}\right) \geq f_{o b j, I_{t h}^{2}}^{\star}\left(\mathcal{Q}_{2}\right)$. By combining the above two inequalities, it is concluded that $f_{o b j, I_{t h}^{1}}^{\star}\left(\mathcal{Q}_{1}\right)=f_{o b j, I_{t h}^{2}}^{\star}\left(\mathcal{Q}_{2}\right)$. Similarly, for an arbitrary threshold $I_{t h} \in\left[I_{t h}^{2}, I_{t h}^{1}\right]$, the same maximum objective value of problem (8) is observed. This phenomenon hints that the

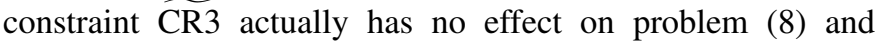
thus can be ignored without loss of optimality. As discussed before, this happens only when $I_{t h}>\tilde{I}_{t h}$, which contradicts to the original condition $0 \leq I_{t h} \leq \tilde{I}_{t h}$. Therefore, the initial assumption of $\mathbf{h}_{M}^{H} \widetilde{\mathbf{W}}_{P, 1}^{\star} \mathbf{h}_{M}+\mathbf{g}_{p}^{H} \widetilde{\mathbf{P}}_{F, 1}^{\star} \mathbf{g}_{p}<I_{t h}^{1}$ is actually invalid, and we must have the optimal solution $\left\{\widetilde{\mathbf{W}}_{P, 1}^{\star}, \widetilde{\mathbf{P}}_{F, 1}^{\star}\right\}$ at the boundary of $\mathbf{h}_{M}^{H} \widetilde{\mathbf{W}}_{P, 1}^{\star} \mathbf{h}_{M}+\mathbf{g}_{p}^{H} \widetilde{\mathbf{P}}_{F, 1}^{\star} \mathbf{g}_{p}=I_{t h}^{1}$ for problem (8) when $0 \leq I_{t h}^{1} \leq \tilde{I}_{t h}$.

\section{B. The proof of Theorem 3}

Firstly, assuming the fixed $\tau$ and $\eta$, we can reexpress the relaxed SRM problem (8) as

$$
\tilde{R}_{S}^{\star}=\max _{0 \leq \tau \leq 1, \eta \geq 1}\left\{\begin{array}{c}
\max _{\widetilde{\mathbf{w}}_{P} \succeq \mathbf{0} \widetilde{\mathbf{P}}_{F} \succeq \mathbf{0}} C_{1} \mathbf{h}_{R}^{H} \widetilde{\mathbf{P}}_{F} \mathbf{h}_{R} \\
\text { s.t. }
\end{array} .\right.
$$

Then the proof for Theorem 3 consists of two steps: In the first step, we prove that for any given $\tau$ and $\eta$, the optimal solution 


$$
\begin{aligned}
& \left(1+\beta^{\star}\right) \mathbf{I}_{N_{F}}+\gamma^{\star} \mathbf{g}_{P} \mathbf{g}_{P}^{H}+\rho^{\star} \mathbf{H}_{E}^{H} \mathbf{R}_{E} \mathbf{H}_{E}-\psi^{\star} C_{1} \mathbf{h}_{R} \mathbf{h}_{R}^{H}-\mathbf{Z}_{F}^{\star}=\mathbf{0} \\
& \lambda^{\star} \mathbf{I}_{N_{P}}+\gamma^{\star} \mathbf{h}_{M} \mathbf{h}_{M}^{H}-\beta^{\star} \xi \mathbf{H}_{F}^{H} \mathbf{H}_{F}-\mathbf{Z}_{P}^{\star}=\mathbf{0} \\
& \mathbf{Z}_{F}^{\star} \widetilde{\mathbf{P}}_{F}^{\star}=\mathbf{0}, \mathbf{Z}_{P}^{\star} \widetilde{\mathbf{W}}_{P}^{\star}=\mathbf{0} \\
& \lambda^{\star}\left(\operatorname{tr}\left(\widetilde{\mathbf{W}}_{P}^{\star}\right)-\tau P_{P}\right)=0 \\
& \beta^{\star}\left(\operatorname{tr}\left(\widetilde{\mathbf{P}}_{F}^{\star}\right)-\xi \operatorname{tr}\left(\mathbf{H}_{F} \widetilde{\mathbf{W}}_{P}^{\star} \mathbf{H}_{F}^{H}\right)-\xi \tau \frac{P_{M}}{N_{M}} \operatorname{tr}\left(\mathbf{G}_{F} \mathbf{G}_{F}^{H}\right)\right)=0 \\
& \gamma^{\star}\left(\mathbf{h}_{M}^{H} \widetilde{\mathbf{W}}_{P}^{\star} \mathbf{h}_{M}+\mathbf{g}_{p}^{H} \widetilde{\mathbf{P}}_{F}^{\star} \mathbf{g}_{p}-I_{t h}\right)=0 \\
& \rho^{\star}\left((1-\eta)(1-\tau)+\operatorname{tr}\left(\mathbf{R}_{E} \mathbf{H}_{E} \widetilde{\mathbf{P}}_{F}^{\star} \mathbf{H}_{E}^{H}\right)\right)=0 \\
& \psi^{\star}\left(C_{1} \mathbf{h}_{R}^{H} \widetilde{\mathbf{P}}_{F}^{\star} \mathbf{h}_{R}-f_{\eta, \tau}\right)=0
\end{aligned}
$$

$\left\{\widetilde{\mathbf{W}}_{P, 1}, \widetilde{\mathbf{P}}_{F, 1}\right\}$ to the inner maximization problem in (30) is of rank-1. In the second step, we show that $\left\{\widetilde{\mathbf{W}}_{P, 1}, \widetilde{\mathbf{P}}_{F, 1}\right\}$ is also optimal to problem (6) (equivalent to the perfect SRM problem (5)), when the same $\tau$ and $\eta$ are adopted. As a result, the equivalence between problem (8) and the perfect SRM problem (5) is established.

Step 1: To prove that the optimal solution to the inner maximization problem in (30) is of rank-1, we firstly consider its equivalent counterpart, i.e. the following power minimization problem

$$
\begin{aligned}
& \min _{\widetilde{\mathbf{W}}_{P} \succeq \mathbf{0}, \widetilde{\mathbf{P}}_{F} \succeq \mathbf{0}} \operatorname{tr}\left(\widetilde{\mathbf{P}}_{F}\right) \\
& \text { s.t. } \widetilde{\mathrm{CR} 1} \sim \widetilde{\mathrm{CR} 4} ; \quad \widetilde{\mathrm{PR} 1}: C_{1} \mathbf{h}_{R}^{H} \widetilde{\mathbf{P}}_{F} \mathbf{h}_{R} \geq f_{\eta, \tau},
\end{aligned}
$$

where $f_{\eta, \tau}$ denotes the maximum objective value of the inner maximization problem in (30). Clearly, problem (29) is still convex with the linear objective function and convex constraints. In fact, the optimal solution of the inner maximization problem in (30) is the same as that of problem (29), which can be proved by contradiction. Firstly, by assuming that the optimal solution to the inner maximization problem in (30) and problem (29) are $\left\{\widetilde{\mathbf{W}}_{P, 1}, \widetilde{\mathbf{P}}_{F, 1}\right\}$ and $\left\{\widetilde{\mathbf{W}}_{P, 2}, \widetilde{\mathbf{P}}_{F, 2}\right\}$, respectively, we readily find that $\left(\widetilde{\mathbf{W}}_{P, 2}, \widetilde{\mathbf{P}}_{F, 2}\right)$ is feasible for the inner maximization problem in (30) and thus we have $C_{1} \mathbf{h}_{R}^{H} \widetilde{\mathbf{P}}_{F, 2} \mathbf{h}_{R} \leq C_{1} \mathbf{h}_{R}^{H} \widetilde{\mathbf{P}}_{F, 1} \mathbf{h}_{R}=f_{\eta, \tau}$. Furthermore, since $\left(\widetilde{\mathbf{W}}_{P, 2}, \widetilde{\mathbf{P}}_{F, 2}\right)$ also satisfies $\widetilde{\mathrm{PR} 1}$, it is inferred that $C_{1} \mathbf{h}_{R}^{H} \widetilde{\mathbf{P}}_{F, 2} \mathbf{h}_{R}=f_{\eta, \tau}$. Clearly, the optimal solution $\left(\widetilde{\mathbf{W}}_{P, 2}, \widetilde{\mathbf{P}}_{F, 2}\right)$ to problem (29) is also optimal to the inner maximization problem in (30). In the sequel, we instead investigate the rank-1 property of the optimal solution to problem (29) through Karush-Kuhn-Tucker (KKT) conditions, which are shown in (30) at the top of this page. Here, $\{\lambda, \beta, \gamma, \rho, \psi\}$ denote non-negative lagrangian multipliers for constraints $\{\widetilde{\mathrm{CR}} 1, \widetilde{\mathrm{CR}} 2, \widetilde{\mathrm{CR}} 3, \widetilde{\mathrm{CR}} 4, \widetilde{\mathrm{PR}} 1\}$ in problem (29), respectively. While $\boldsymbol{Z}_{P} \succeq \mathbf{0}$ and $\boldsymbol{Z}_{F} \succeq \mathbf{0}$ are the lagrangian multipliers corresponding to $\widetilde{W}_{P} \succeq \mathbf{0}$ and $\widetilde{\boldsymbol{P}}_{F} \succeq \mathbf{0}$, respectively. Based on (30a) and (30c), we have $\left(\left(1+\beta^{\star}\right) \mathbf{I}_{N_{F}}+\gamma^{\star} \mathbf{g}_{P} \mathbf{g}_{P}^{H}+\rho^{\star} \mathbf{H}_{E}^{H} \mathbf{R}_{E} \mathbf{H}_{E}\right) \widetilde{\mathbf{P}}_{F}^{\star}=$ $\psi^{\star} C_{1} \mathbf{h}_{R} \mathbf{h}_{R}^{H} \widetilde{\mathbf{P}}_{F}^{\star}$, implying that

$$
\begin{aligned}
& \operatorname{rank}\left(\left(\left(1+\beta^{\star}\right) \mathbf{I}_{N_{F}}+\gamma^{\star} \mathbf{g}_{P} \mathbf{g}_{P}^{H}+\rho^{\star} \mathbf{H}_{E}^{H} \mathbf{R}_{E} \mathbf{H}_{E}\right) \widetilde{\mathbf{P}}_{F}^{\star}\right) \\
& =\operatorname{rank}\left(\widetilde{\mathbf{P}}_{F}^{\star}\right)=\operatorname{rank}\left(\psi^{\star} C_{1} \mathbf{h}_{R} \mathbf{h}_{R}^{H} \widetilde{\mathbf{P}}_{F}^{\star}\right) \leq 1,
\end{aligned}
$$

where the first equality in (31) is due to $\left(1+\beta^{\star}\right) \mathbf{I}_{N_{F}}+$ $\gamma^{\star} \mathbf{g}_{P} \mathbf{g}_{P}^{H}+\rho^{\star} \mathbf{H}_{E}^{H} \mathbf{R}_{E} \mathbf{H}_{E} \succ \mathbf{0}$.
According to (31), the rank-1 optimal $\widetilde{\mathbf{P}}_{F}^{\star}$ to problem (29) is proved. As for the optimal $\widetilde{\mathbf{W}}_{P}^{\star}$, we refer to (30b) and (30c) to obtain

$$
\lambda^{\star} \mathbf{I}_{N_{P}}-\widetilde{\mathbf{H}}^{\star}=\mathbf{Z}_{P}^{\star} \succeq \mathbf{0}, \quad\left(\lambda^{\star} \mathbf{I}_{N_{P}}-\widetilde{\mathbf{H}}^{\star}\right) \widetilde{\mathbf{W}}_{P}^{\star}=\mathbf{0},
$$

where $\widetilde{\mathbf{H}}^{\star}=\beta^{\star} \xi \mathbf{H}_{F}^{H} \mathbf{H}_{F}-\gamma^{\star} \mathbf{h}_{M} \mathbf{h}_{M}^{H}$. Observing from (32), if $\lambda^{\star}=0$, then we have $\beta^{\star} \xi \mathbf{H}_{F}^{H} \mathbf{H}_{F} \preceq \gamma^{\star} \mathbf{h}_{M} \mathbf{h}_{M}^{H}$, for which $\beta^{\star}=0$ is derived since $\operatorname{rank}\left(\xi \mathbf{H}_{F}^{H} \mathbf{H}_{F}\right)>\operatorname{rank}\left(\mathbf{h}_{M} \mathbf{h}_{M}^{H}\right)=1$ is implied by our system setting. The optimal $\widetilde{\mathbf{W}}_{P}^{\star}$ is then derived as $\mathbf{h}_{M} \mathbf{h}_{M}^{H} \widetilde{\mathbf{W}}_{P}^{\star}=0$. Clearly, there must exist a rank-1 optimal $\widehat{\mathbf{W}}_{P}^{\star}$ in the null space of $\mathbf{h}_{M}$. However, if $\lambda^{\star} \geq 0$, then we have the eigenvalue decomposition (EVD) of $\widetilde{\mathbf{H}}^{\star}$ as $\widetilde{\mathbf{H}}^{\star}=\widetilde{\mathbf{U}}_{H} \widetilde{\boldsymbol{\Lambda}}_{H} \widetilde{\mathbf{U}}_{H}^{H}$, where the maximum eigenvalue $\tilde{\lambda}_{H, \text { max }}$ in the diagonal matrix $\widetilde{\boldsymbol{\Lambda}}_{H}$ must be positive. Otherwise, $\beta^{\star} \xi \mathbf{H}_{F}^{H} \mathbf{H}_{F} \preceq \gamma^{\star} \mathbf{h}_{M} \mathbf{h}_{M}^{H}$ is implied and only obtained at $\beta^{\star}=0$ as mentioned above. Based on (32), we then have $\widetilde{\mathbf{W}}_{P}^{\star}=\mathbf{0}$ since $\lambda^{\star}>0$, which contradicts with (30d). So $\lambda^{\star}>0$ yields $\tilde{\lambda}_{H, \text { max }}>0$ and thus we have $\lambda^{\star} \mathbf{I}_{N_{P}}-\widetilde{\mathbf{H}}^{\star}=\widetilde{\mathbf{U}}_{H}\left(\lambda^{\star} \mathbf{I}_{N_{P}}-\widetilde{\boldsymbol{\Lambda}}_{H}\right) \widetilde{\mathbf{U}}_{H}^{H} \succeq \mathbf{0}$. Further, to ensure $\widetilde{\mathbf{W}}_{P}^{\star} \neq \mathbf{0}$ in (32), it is easily inferred that $\lambda^{\star}=\tilde{\lambda}_{H, \max }$ and thus $\widetilde{\mathbf{W}}_{P}^{\star}=c \mathbf{u}_{P} \mathbf{u}_{P}^{H}$ is obtained, where $\mathbf{u}_{P}$ is the unit-norm eigenvector of $\widetilde{\mathbf{H}}^{\star}$ corresponding to $\lambda_{H, \max }$. Moreover, since $\lambda^{\star}=\tilde{\lambda}_{H, \text { max }}>0$, it yields that $\operatorname{tr}\left(\widetilde{\mathbf{W}}_{P}^{\star}\right)=\tau P_{P}$ and thus $\widetilde{\mathbf{W}}_{P}^{\star}=\tau P_{P} \mathbf{u}_{P} \mathbf{u}_{P}^{H}$ can be obtained from (30d). Both cases of $\lambda^{\star}$ demonstrate that the optimal $\widetilde{\mathbf{W}}_{P}^{\star}$ to problem (29) is of rank-1. Overall, the rank-1 optimal solution $\left\{\widetilde{\mathbf{W}}_{P}, \widetilde{\mathbf{P}}_{F}\right\}$ of the inner maximization problem in (30) is proved.

Step 2: Firstly, we assume that the optimal solution of the inner maximization problem in (30) (equivalent to problem ) and problem (6) with the same $\{\eta, \tau\}$ are $\left\{\widetilde{\mathbf{W}}_{P, 1}, \widetilde{\mathbf{P}}_{F, 1}\right\}$ and $\left\{\widetilde{\mathbf{W}}_{P, 0}, \widetilde{\mathbf{P}}_{F, 0}\right\}$, respectively. The corresponding objective function is defined as $f\left(\widetilde{\mathbf{W}}_{P}, \widetilde{\mathbf{P}}_{F}\right)$. Based on Lemma 1 , for any given $\eta$ and $\tau$, the relaxed SRM problem (8) actually has a larger feasible solution region than problem (6), hence, we have $f\left(\widetilde{\mathbf{W}}_{P, 1}, \widetilde{\mathbf{P}}_{F, 1}\right) \geq f\left(\widetilde{\mathbf{W}}_{P, 0}, \widetilde{\mathbf{P}}_{F, 0}\right)$. Furthermore, since both $\widetilde{\mathbf{W}}_{P, 1}$, and $\widetilde{\mathbf{P}}_{F, 1}$ are of rank-1 as proved in Step 1, we also find that $\left\{\widetilde{\mathbf{W}}_{P, 1}, \widetilde{\mathbf{P}}_{F, 1}\right\}$ is feasible to problem (6), which implies that $f\left(\widetilde{\mathbf{W}}_{P, 1}, \widetilde{\mathbf{P}}_{F, 1}\right) \leq f\left(\widetilde{\mathbf{W}}_{P, 0}, \widetilde{\mathbf{P}}_{F, 0}\right)$. Combining the above two inequalities, we finally have $f\left(\widetilde{\mathbf{W}}_{P, 1}, \widetilde{\mathbf{P}}_{F, 1}\right)=$ $f\left(\widetilde{\mathbf{W}}_{P, 0}, \widetilde{\mathbf{P}}_{F, 0}\right)$.

Given any $\tau$ and $\eta$, it is concluded that such a twotupple $\left\{\widetilde{\mathbf{W}}_{P, 1}, \widetilde{\mathbf{P}}_{F, 1}\right\}$ from the relaxed SRM problem (8) (inner maximization problem in (30)) is optimal to problem (6). Notice that problem (6) with the optimal $\tau^{\star}$ and $\eta^{\star}$ is 
equivalent to the original SRM problem (5), Therefore, the equivalence between problem (8) and the PSRM problem (5) is established as that in Theorem 3.

\section{APPENDIX B}

Notice that this proof follows the same logic as that provided for Theorem 3. Firstly, assuming the fixed $\tau$ and $\eta$, we can reexpress the relaxed robust SRM problem (17) as

$$
\tilde{R}_{S, R o}^{\star}=\max _{\substack{0 \leq \tau \leq 1 \\
\eta \geq 1}}\left\{\begin{array}{l}
\max _{\widetilde{\mathbf{W}}_{P} \succeq \mathbf{0 ,} \widetilde{\mathbf{P}}_{F} \succeq \mathbf{0}} C_{1} \mathbf{h}_{R}^{H} \widetilde{\mathbf{P}}_{F} \mathbf{h}_{R} \\
\text { s.t. } \widetilde{\mathrm{CR} 1} \sim \widetilde{\mathrm{CR} 3} \\
\widetilde{\mathrm{CR} 5}: \widetilde{\mathbf{I}}_{E}-\widetilde{\mathbf{R}}_{E}\left(\mathbf{I}_{N} \otimes \widetilde{\mathbf{P}}_{F}\right) \widetilde{\mathbf{R}}_{E}^{H} \succeq \mathbf{0}
\end{array}\right.
$$

where $\widetilde{\mathbf{I}}_{E}=\left[\begin{array}{cc}u \mathbf{I}_{N} & \mathbf{0} \\ \mathbf{0} & \eta-1-\mu \xi_{f}^{2}\end{array}\right]$ and $\quad \widetilde{\mathbf{R}}_{E}=$ $\left[\begin{array}{c}\mathbf{R}_{E}^{\frac{1}{2} T} \otimes \mathbf{I}_{N} \\ \hat{\mathbf{h}}_{E}^{H}\left(\mathbf{R}_{E}^{\frac{1}{2} T} \otimes \mathbf{I}_{N}\right)\end{array}\right]$. The proof of Theorem 4 is similar to that of Theorem 3 and also consists of the following two steps. In the first step, we prove that for any given $\tau$ and $\eta$, the rank-1 optimal solution $\left\{\widetilde{\mathbf{W}}_{P}, \widetilde{\mathbf{P}}_{F}\right\}$ of the inner maximization problem in (33) is obtained. In the second step, we show that such an $\left\{\widetilde{\mathbf{W}}_{P}, \widetilde{\mathbf{P}}_{F}\right\}$ is also optimal to the WSRM problem (12). As a result, the equivalence between the relaxed WSRM problem (17) and the original WSRM problem (12) are established. Similarly, for proving the rank-1 nature of the optimal solution to the inner maximization problem in (33), we consider the corresponding power minimization problem given $\tau$ and $\eta$ as

$$
\begin{aligned}
& \min _{\widetilde{\mathbf{W}}_{P} \succeq \mathbf{0}, \widetilde{\mathbf{P}}_{F} \succeq \mathbf{0}} \operatorname{tr}\left(\widetilde{\mathbf{P}}_{F}\right) \\
& \text { s.t. } \widetilde{\mathrm{CR} 1} \sim \widetilde{\mathrm{CR} 3}, \widetilde{\mathrm{CR} 5}, \widetilde{\mathrm{PR}} 1: C_{1} \mathbf{h}_{R}^{H} \widetilde{\mathbf{P}}_{F} \mathbf{h}_{R} \geq \hat{f}_{\eta, \tau},
\end{aligned}
$$

where $\hat{f}_{\eta, \tau}$ denotes the optimal objective value of the inner maximization problem in (33) given any $\tau$ and $\eta$. It is readily observed that problem (34) is also convex. Following the same philosophy in Step 1 of Appendix A-B, we readily verify that the optimal solution of problem (34) is also optimal to the inner maximization problem in (33). In the sequel, we aim to show that the optimal solution $\left\{\widetilde{\mathbf{W}}_{P}, \widetilde{\mathbf{P}}_{F}\right\}$ of problem (34) for any fixed $\tau$ and $\eta$ are of rank-1 through KKT conditions, which are

$$
\begin{aligned}
& \left(1+\beta^{\star}\right) \mathbf{I}_{N_{F}}+\gamma^{\star} \mathbf{g}_{P} \mathbf{g}_{P}^{H}+\rho^{\star} \mathbf{H}_{E}^{H} \mathbf{R}_{E} \mathbf{H}_{E} \\
& +\sum_{i=1}^{N_{E}} \widetilde{\mathbf{R}}_{E, i}^{H} \mathbf{Z}_{R} \widetilde{\mathbf{R}}_{E, i}-\psi^{\star} C_{1} \mathbf{h}_{R} \mathbf{h}_{R}^{H}-\mathbf{Z}_{F}^{\star}=\mathbf{0}, \\
& \mathbf{Z}_{R}^{\star}\left(\widetilde{\mathbf{I}}_{E}-\widetilde{\mathbf{R}}_{E}\left(\mathbf{I}_{N} \otimes \widetilde{\mathbf{P}}_{F}\right) \widetilde{\mathbf{R}}_{E}^{H}\right)=\mathbf{0} \\
& (30 \mathrm{~b}) \sim(30 \mathrm{f}),(30 \mathrm{~h})
\end{aligned}
$$

where $Z_{R} \succeq \mathbf{0}$ is lagrangian multiplier for $\widetilde{\mathrm{CR} 5}$. Based on (35a) and (30c), we have

$$
\begin{aligned}
& \operatorname{rank}\left[\left(\left(1+\beta^{\star}\right) \mathbf{I}_{N_{F}}+\gamma^{\star} \mathbf{g}_{P} \mathbf{g}_{P}^{H}+\rho^{\star} \mathbf{H}_{E}^{H} \mathbf{R}_{E} \mathbf{H}_{E}\right.\right. \\
& \left.\left.\quad+\sum_{i=1}^{N_{E}} \widetilde{\mathbf{R}}_{E, i}^{H} \mathbf{Z}_{R} \widetilde{\mathbf{R}}_{E, i}\right) \widetilde{\mathbf{P}}_{F}^{\star}\right] \\
& =\operatorname{rank}\left(\widetilde{\mathbf{P}}_{F}^{\star}\right)=\operatorname{rank}\left(\psi^{\star} C_{1} \mathbf{h}_{R} \mathbf{h}_{R}^{H} \widetilde{\mathbf{P}}_{F}^{\star}\right) \leq 1 .
\end{aligned}
$$

It is clear from (36) that the optimal $\widetilde{\mathbf{P}}_{F}^{\star}$ to problem (34) is of rank-1. In addition, since the $\widetilde{\mathbf{W}}_{P}$ related KKT conditions of problem (34) are the same as that of problem (29). Let's refer to Step 1 of Appendix C to prove the rank-1 optimal $\widetilde{\mathbf{W}}_{P}^{\star}$ to problem (34). As such, the rank-1 optimal solution $\left\{\widetilde{\mathbf{P}}_{F}^{\star}, \widetilde{\mathbf{W}}_{P}^{\star}\right\}$ to problem (34) are finally verified.

\section{APPENDIX C}

According to [32] and following the same argument as Step 1 of Appendix A-B, the outage-constrained SRM problem (23) given any $\{\tau, \mathbf{S}\}$ is equivalent to the following power minimization problem

$$
\min _{\widetilde{\mathbf{W}}_{P} \succeq \mathbf{0}, \widetilde{\mathbf{P}}_{F} \succeq \mathbf{0}} \operatorname{tr}\left(\widetilde{\mathbf{P}}_{F}\right), \quad \text { s.t. } \widetilde{\mathrm{CR} 1} \sim \widetilde{\mathrm{CR} 3},\left.\widetilde{\mathrm{CR} 6}\right|_{R_{S}=R_{S}^{1}},
$$

where $R_{S}^{1}$ denotes the optimal objective value of the problem (23) with fixed $\tau$ and $\mathbf{S}$. In other words, the optimal solution $\left\{\widetilde{\mathbf{P}}_{F}^{\star}, \widetilde{\mathbf{W}}_{P}^{\star}\right\}$ to problem (37) is also optimal to problem (23) given the same $\tau$ and $\mathbf{S}$. In the sequel, we firstly prove the rank-1 optimal $\widetilde{\mathbf{P}}_{F}^{\star}$ to problem (37). First of all, we define the projection matrix $\mathbf{T}$ of the vector $\widetilde{\mathbf{P}}_{F}^{\frac{1}{2}{ }^{\star}} \mathbf{h}_{R}$ as $\mathbf{T}=$ $\frac{\widetilde{\mathbf{P}}_{F}^{\frac{1}{2} \star} \mathbf{h}_{R} \mathbf{h}_{R}^{H} \widetilde{\mathbf{P}}_{F}^{\frac{1}{2} \star}}{\left\|\mathbf{h}_{R}^{H} \widetilde{\mathbf{P}}_{F}^{\frac{1}{2} \star}\right\|^{2}}$, then a novel rank-1 solution $\widehat{\mathbf{P}}_{F}^{\star}=\widetilde{\mathbf{P}}_{F}^{\frac{1}{2} \star} \mathbf{T} \widetilde{\mathbf{P}}_{F}^{\frac{1}{2} \star}$ is available and satisfies

$$
\operatorname{tr}\left(\widehat{\mathbf{P}}_{F}^{\star}\right)-\operatorname{tr}\left(\widetilde{\mathbf{P}}_{F}^{\star}\right)=\operatorname{tr}\left(\widetilde{\mathbf{P}}_{F}^{\frac{1}{2} \star}(\mathbf{T}-\mathbf{I}) \widetilde{\mathbf{P}}_{F}^{\frac{1}{2} \star}\right) \leq 0 .
$$

The formulation (38) yields $\operatorname{tr}\left(\widehat{\mathbf{P}}_{F}^{\star}\right) \leq \operatorname{tr}\left(\widetilde{\mathbf{P}}_{F}^{\star}\right)$, which hints that the objective value of problem (23) is non-increasing while still satisfying constraints $\widehat{\mathrm{CR} 1} \sim \widetilde{\mathrm{CR} 2}$. Moreover, observing from CR6, we have

$$
\begin{aligned}
& \log _{2}\left(1+\frac{C_{1} \mathbf{h}_{R}^{H} \widehat{\mathbf{P}}_{F}^{\star} \mathbf{h}_{R}}{1-\tau}\right) \\
& =\log _{2}\left(1+\frac{C_{1}\left|\mathbf{h}_{R}^{H} \widetilde{\mathbf{P}}_{F}^{\star} \mathbf{h}_{R}\right|^{2}}{(1-\tau)\left\|\widetilde{\mathbf{P}}_{F}^{\frac{1}{2} \star} \mathbf{h}_{R}\right\|^{2}}\right)=\log _{2}\left(1+\frac{C_{1} \mathbf{h}_{R}^{H} \widetilde{\mathbf{P}}_{F}^{\star} \mathbf{h}_{R}}{1-\tau}\right)
\end{aligned}
$$

as well (40) (shown at the top of the next page). Based on (39) and (40), it yields

$$
\begin{aligned}
& \log _{2}\left(1+\frac{C_{1} \mathbf{h}_{R}^{H} \widehat{\mathbf{P}}_{F}^{\star} \mathbf{h}_{R}}{1-\tau}\right)-\log _{2} \operatorname{det}\left(\mathbf{I}_{N_{E}}+\frac{\mathbf{R}_{E} \mathbf{H}_{E} \widehat{\mathbf{P}}_{F}^{\star} \mathbf{H}_{E}^{H}}{1-\tau}\right) \\
& \geq \log _{2}\left(1+\frac{C_{1} \mathbf{h}_{R}^{H} \widetilde{\mathbf{P}}_{F}^{\star} \mathbf{h}_{R}}{1-\tau}\right)-\log _{2} \operatorname{det}\left(\mathbf{I}_{N_{E}}+\frac{\mathbf{R}_{E} \mathbf{H}_{E} \widetilde{\mathbf{P}}_{F}^{\star} \mathbf{H}_{E}^{H}}{1-\tau}\right),
\end{aligned}
$$

which means that the constraint $\widetilde{\mathrm{CR} 6}$ still holds by using the novel solution $\widehat{\mathbf{P}}_{F}^{\star}$. Similarly, by referring to (38) and the following inequality

$$
\begin{aligned}
\mathbf{g}_{p}^{H} \widehat{\mathbf{P}}_{F}^{\star} \mathbf{g}_{p} & =\mathbf{g}_{p}^{H} \widetilde{\mathbf{P}}_{F}^{\frac{1}{2} \star} \mathbf{T} \widetilde{\mathbf{P}}_{F}^{\frac{1}{2} \star} \mathbf{g}_{p} \\
& =\frac{\left|\mathbf{g}_{p}^{H} \widetilde{\mathbf{P}}_{F}^{\frac{1}{2} \star} \widetilde{\mathbf{P}}_{F}^{\frac{1}{2} \star} \mathbf{h}_{R}\right|^{2}}{\left\|\widetilde{\mathbf{P}}_{F}^{\frac{1}{2} \star} \mathbf{h}_{R}\right\|^{2}} \leq \mathbf{g}_{p}^{H} \widetilde{\mathbf{P}}_{F}^{\star} \mathbf{g}_{p},
\end{aligned}
$$

the constraint $\widetilde{\mathrm{CR} 3}$ can also be satisfied with $\left\{\widehat{\mathbf{P}}_{F}^{\star}, \widetilde{\mathbf{W}}{ }_{P}^{\star}\right\}$. This phenomenon indicates that the novel solution $\left\{\widehat{\mathbf{P}}_{F}^{\star}, \widetilde{\mathbf{W}}_{P}^{\star}\right\}$ is also feasible to problem (37) and may even realize a lower objective value than $\left\{\widetilde{\mathbf{P}}_{F}^{\star}, \widetilde{\mathbf{W}}_{P}^{\star}\right\}$. As a result, we conclude 


$$
\begin{aligned}
& \log _{2} \operatorname{det}\left(\mathbf{I}_{N_{E}}+\frac{\mathbf{R}_{E} \mathbf{H}_{E} \widehat{\mathbf{P}}_{F}^{\star} \mathbf{H}_{E}^{H}}{1-\tau}\right)=\log _{2}\left(\mathbf{I}_{N_{E}}+\frac{\mathbf{R}_{E} \mathbf{H}_{E} \widetilde{\mathbf{P}}_{F}^{\frac{1}{2} \star} \mathbf{T} \widetilde{\mathbf{P}}_{F}^{\frac{1}{2} \star} \mathbf{H}_{E}^{H}}{(1-\tau)}\right) \\
& =\log _{2}\left(1+\frac{\mathbf{h}_{R}^{H} \widetilde{\mathbf{P}}_{F}^{\frac{1}{2} \star}\left(\widetilde{\mathbf{P}}_{F}^{\frac{1}{2} \star} \mathbf{H}_{E}^{H} \mathbf{R}_{E} \mathbf{H}_{E} \widetilde{\mathbf{P}}_{F}^{\frac{1}{2} \star}\right) \widetilde{\mathbf{P}}_{F}^{\frac{1}{2} \star} \mathbf{h}_{R}}{(1-\tau)\left\|\mathbf{h}_{R}^{H} \widetilde{\mathbf{P}}_{F}^{\frac{1}{2} \star}\right\|^{2}}\right) \leq \log _{2}\left(1+\frac{\lambda_{\max }\left(\widetilde{\mathbf{P}}_{F}^{\frac{1}{2} \star} \mathbf{H}_{E}^{H} \mathbf{R}_{E} \mathbf{H}_{E} \widetilde{\mathbf{P}}_{F}^{\frac{1}{2} \star}\right)}{1-\tau}\right) \\
& \leq \log _{2} \operatorname{det}\left(\mathbf{I}_{N_{F}}+\frac{\widetilde{\mathbf{P}}_{F}^{\frac{1}{2} \star} \mathbf{H}_{E}^{H} \mathbf{R}_{E} \mathbf{H}_{E} \widetilde{\mathbf{P}}_{F}^{\frac{1}{2} \star}}{1-\tau}\right)=\log _{2} \operatorname{det}\left(\mathbf{I}_{N_{E}}+\frac{\mathbf{R}_{E} \mathbf{H}_{E} \widetilde{\mathbf{P}}_{F}^{\star} \mathbf{H}_{E}^{H}}{1-\tau}\right) .
\end{aligned}
$$

that the optimal $\mathbf{P}_{F}^{\star}$ to problem (37) (equivalent to the outageconstrained SRM problem (23)) must be of rank-1. Since the $\widetilde{\mathbf{W}}_{P}$ related KKT conditions of problem (23) are also the same as that of problem (29), the proof for the rank-1 optimal $\widetilde{\mathbf{W}}_{P}$ to problem (29) in Appendix A-B can still be applied to problem (23). Due to space limitation, the detailed proof is omitted here. Overall, the rank-1 optimal solution $\widetilde{\mathbf{P}}_{F}^{\star}, \widetilde{\mathbf{W}}_{P}^{\star}$ to the outage-constrained SRM problem (23) is proved.

\section{APPENDIX D}

Recalling the proof in Step 1 of Appendix A, for any fixed $\tau$, we readily infer that the AN aided problem (27) is equivalent to the following power minimization problem

$$
\begin{aligned}
\min _{\tau, \widetilde{\mathbf{W}}_{P} \succeq \mathbf{0}, \widetilde{\mathbf{P}}_{F} \succeq \mathbf{0}, \widetilde{\mathbf{\Sigma}}_{U} \succeq \mathbf{0}} \operatorname{tr}\left(\widetilde{\mathbf{P}}_{F}\right) \\
\text { s.t. } \widetilde{\mathrm{CR} 1}, \widetilde{\mathrm{AR} 3}, \widetilde{\mathrm{CR} 3}, \widetilde{\mathrm{AR} 1}: \operatorname{tr}\left(\widetilde{\mathbf{\Sigma}}_{U}\right) \geq f_{A}, \\
\\
\widetilde{\mathrm{AR} 2}: \operatorname{tr}\left(C_{1} \mathbf{h}_{R}^{H} \widetilde{\mathbf{P}}_{F} \mathbf{h}_{R}\right) \geq C_{2},
\end{aligned}
$$

where $f_{A}$ denotes the optimal objective value of problem (27) and $C_{2}=(1-\tau)\left(2^{\frac{R_{t h}}{(1-\tau)}}-1\right)$. To demonstrate the rank-1 optimal solution $\left\{\widetilde{\mathbf{W}}_{P}, \widetilde{\mathbf{P}}_{F}, \widetilde{\mathbf{\Sigma}}_{U}\right\}$ to problem (43), we formulate the corresponding KKT conditions as

$$
\begin{aligned}
& \left(1+\beta^{\star}\right) \mathbf{I}_{N_{F}}+\gamma^{\star} \mathbf{g}_{p} \mathbf{g}_{p}^{H}-\psi^{\star} C_{1} \mathbf{h}_{R} \mathbf{h}_{R}^{H}-\mathbf{Z}_{F}^{\star}=\mathbf{0}, \\
& \left(\beta^{\star}-\rho^{\star}\right) \mathbf{I}_{N_{F}}+\gamma^{\star} \mathbf{g}_{p} \mathbf{g}_{p}^{H}-\mathbf{Z}_{U}^{\star}=\mathbf{0}, \\
& \mathbf{Z}_{U}^{\star} \widetilde{\boldsymbol{\Sigma}}_{U}^{\star}=\mathbf{0},(30 \mathrm{~b}) \sim(30 \mathrm{f}),\left.\quad(30 \mathrm{~h})\right|_{f_{\eta, \tau}=C_{2}},
\end{aligned}
$$

where $\left\{\rho^{\star}, \psi^{\star}, \beta^{\star}\right\}, \lambda^{\star}$ and $\gamma^{\star}$ are the optimal lagrangian multipliers associated with constraints $\widetilde{\mathrm{AR}} 1 \sim \widetilde{\mathrm{AR}} 3, \overline{\mathrm{CR}} 1$ and $\widetilde{\mathrm{CR} 3}$, respectively, while $\mathbf{Z}_{U}^{\star} \succeq \mathbf{0}$ is the optimal lagrangian multiplier for $\widetilde{\boldsymbol{\Sigma}}_{U} \succeq \mathbf{0}$. It is readily observed from (44a) and (30c) that

$$
\begin{aligned}
& \operatorname{rank}\left(\left[\left(1+\beta^{\star}\right) \mathbf{I}_{N_{F}}+\gamma^{\star} \mathbf{g}_{P} \mathbf{g}_{P}^{H}\right] \widetilde{\mathbf{P}}_{F}^{\star}\right)=\operatorname{rank}\left(\widetilde{\mathbf{P}}_{F}^{\star}\right) \\
& \quad=\operatorname{rank}\left(\psi^{\star} C_{1} \mathbf{h}_{R} \mathbf{h}_{R}^{H} \widetilde{\mathbf{P}}_{F}^{\star}\right) \leq 1,
\end{aligned}
$$

which implies that the optimal $\widetilde{\mathbf{P}}_{F}^{\star}$ to problem (43) is of rank1. Additionally, based on (44b) and (44c), we also find that $\beta^{\star}-\rho^{\star} \geq 0$ for guaranteeing $\mathbf{Z}_{U}^{\star} \succeq \mathbf{0}$. To be specific, when $\beta^{\star}-\rho^{\star}=0$, we have $\gamma^{\star} \mathbf{g}_{p} \mathbf{g}_{p}^{H} \boldsymbol{\Sigma}_{U}^{\star}=\mathbf{0}$. Clearly, there must exist a rank-1 optimal $\widetilde{\boldsymbol{\Sigma}}_{U}^{\star}$ within the null space of $\mathbf{g}_{p}^{H}$. While for $\beta^{\star}-\rho^{\star}>0, \mathbf{Z}_{U}^{\star} \succ \mathbf{0}$ and $\widetilde{\boldsymbol{\Sigma}}_{U}^{\star}=\mathbf{0}$ are obtained according to (44c). Based on above discussion, the rank-1 optimal $\widetilde{\Sigma}_{U}^{\star}$ to problem (43) can be proved. Without loss of generality, the $\widetilde{\mathbf{W}}_{P}$ related constraints of problem (43) are also identical to that of problem (29). Therefore, the rank-1 nature of the optimal $\widetilde{\mathbf{W}}_{P}^{\star}$ to problem (43) can be verified. Considering the equivalence between problems (43) and (27), we finally conclude that the optimal solution $\left\{\widetilde{\mathbf{W}}_{P}, \widetilde{\mathbf{P}}_{F}, \widetilde{\mathbf{\Sigma}}_{U}\right\}$ of problem (27) is also of rank-1.

\section{APPENDIX E}

Since it has been proved that the globally optimal or highquality suboptimal FBS information covariance matrices $\mathbf{P}_{F}$ for all studied problems, namely (5), (12), (18) and (26), are all of rank-1, accordingly, we can define the optimal $\mathbf{P}_{F}^{\star}=$ $\lambda_{P} \mathbf{p}_{F} \mathbf{p}_{F}^{H}$ with $\left\|\mathbf{p}_{F}\right\|_{F}=1$ and $\lambda_{P}>0$ for all these problems. Furthermore, by substituting $\mathbf{P}_{F}^{\star}=\lambda_{P} \mathbf{p}_{F} \mathbf{p}_{F}^{H}$ into the secrecy rate expression in (4), it yields

$$
\begin{aligned}
& R_{S}=(1-\tau) \log _{2}\left(1+C_{1} \lambda_{P} \mathbf{h}_{R}^{H} \boldsymbol{p}_{F} \boldsymbol{p}_{F}^{H} \mathbf{h}_{R}\right) \\
& -(1-\tau) \log _{2} \operatorname{det}\left(\mathbf{I}_{N_{E}}+\mathbf{R}_{E} \mathbf{H}_{E} \mathbf{P}_{F} \mathbf{H}_{E}^{H}\right) \\
& =(1-\tau) \log _{2}\left(1+\frac{C_{1}\left|\mathbf{h}_{R}^{H} \boldsymbol{p}_{F}\right|^{2}-\left\|\mathbf{R}_{E}^{\frac{1}{2}} \mathbf{H}_{E} \mathbf{p}_{F}\right\|_{F}^{2}}{\frac{1}{\lambda_{P}}+\left\|\mathbf{R}_{E}^{\frac{1}{2}} \mathbf{H}_{E} \mathbf{p}_{F}\right\|_{F}^{2}}\right) .
\end{aligned}
$$

Notice that our work aims to optimize $\boldsymbol{P}_{F}$ for achieving the maximum non-negative secrecy rate $R_{S}$, so we must have $C_{1}\left|\mathbf{h}_{R}^{H} \boldsymbol{p}_{F}\right|^{2} \geq\left\|\mathbf{R}_{E}^{\frac{1}{2}} \mathbf{H}_{E} \mathbf{p}_{F}\right\|_{F}^{2}$ at the optimal $\mathbf{P}_{F}^{\star}=\lambda_{P} \mathbf{p}_{F} \mathbf{p}_{F}^{H}$. Based on this, it can be found from (46) that $R_{S}$ is a monotonically non-decreasing function of $\lambda_{P}$. Additionally, when the cross-interference constraint CR3 is inactive for each problem, it is clear that $\lambda_{P}$ is only subject to the constraint CR2 for problems (5), (12), (18), or constraints AR2 and AR3 for problem (26). Based on (46) and problem (26), we readily infer that both CR2 and AR3 are active at the optimal $\mathbf{P}_{F}^{\star}$ for maximizing secrecy rate $R_{S}$ and artificial noise power, respectively. Inspired by this conclusion, we further formulate the common subproblem for optimizing PB energy covariance matrices $\mathbf{W}_{P}$ in different cases of FBS-EVE CSI with the inactive $\mathrm{CR} 3$ as

$$
\begin{aligned}
& \max _{\tau, \mathbf{W}_{P} \succeq \mathbf{0}} \tau \xi \operatorname{tr}\left(\mathbf{H}_{F} \mathbf{W}_{P} \mathbf{H}_{F}^{H}\right) \\
& \text { s.t. } \quad \mathrm{CR} 1: 0 \leq \tau \leq 1, \operatorname{tr}\left(\mathbf{W}_{P}\right) \leq P_{P} .
\end{aligned}
$$

According to [35, Lemma H.1.h], we readily derive the closed-form solution $\mathbf{W}_{P}^{\star}$ to problem (47) as $\mathbf{W}_{P}^{\star}=$ $P_{P} \mathbf{w}_{P} \mathbf{w}_{P}^{H}$ with $\mathbf{w}_{P}=\nu_{\max }\left(\mathbf{H}_{F}^{H} \mathbf{H}_{F}\right)$.

\section{REFERENCES}

[1] M. Shafi et al.,"5G: A tutorial overview of standards, trials, challenges, deployment, and practice," IEEE J. Select. Areas Commun., vol. 35, no. 6, pp. 1201-1221, Jun. 2017. 
[2] F. Boccardi, R. Heath, A. Lozano, T. Marzetta, and P. Popovski, "Five disruptive technology directions for 5G," IEEE Commun. Mag., vol. 52, no. 2, pp. 74-80, Feb. 2014.

[3] E. Hossain, M. Rasti, H. Tabassum, and A. Abdelnasser, "Evolution toward 5G multi-tier cellular wireless networks: An interference management perspective," IEEE Wireless Commun., vol. 21, no. 3, pp. 118 127, Jun. 2014.

[4] G. Zhao, S. Chen, L. Zhao and L. Hanzo,"Energy-spectral-efficiency analysis and optimization of heterogeneous cellular networks: A largescale user-behavior perspective," IEEE Trans. Veh. Techno., vol. 67, no. 5, pp. 4098-4112, May 2018.

[5] H. S. Jo, Y. J. Sang, P. Xia, and J. G. Andrews, "Heterogeneous cellular networks with flexible cell association: A comprehensive downlink SINR analysis," IEEE Trans. Wireless Commun., vol. 11, no. 10, pp. 3484 3495, Oct. 2012.

[6] A. Damnjanovic et al.,"A survey on 3GPP heterogeneous networks," IEEE Wireless Commun., vol. 18, no. 3, pp. 10-21, Jun. 2011.

[7] S. Gong, S. Ma, C. Xing and G. Yang,"Optimal beamforming and time allocation for partially wireless powered sensor networks with downlink SWIPT," IEEE Trans. Signal Process., vol. 67, no. 12, pp. 3197-3212, Jun. 2019

[8] J. Hu, K. Yang, G. Wen and L. Hanzo,"Integrated data and energy communication network: A comprehensive survey," IEEE Commun. Surveys Tuts., vol. 20, no. 4, pp. 3169-3219, Fourthquarter 2018.

[9] M. Zhao, Q. Shi, Y. Cai and M. Zhao,"Joint transceiver design for fullduplex cloud radio access networks with SWIPT," IEEE Trans. Wireless Commun., vol. 16, no. 9, pp. 5644-5658, Sept. 2017.

[10] R. Zhang, R. G. Maunder and L. Hanzo, "Wireless information and power transfer: from scientific hypothesis to engineering practice," IEEE Commun. Mag., vol. 53, no. 8, pp. 99-105, Aug. 2015.

[11] H. Tabassum and E. Hossain, "On the deployment of energy sources in wireless-powered cellular networks," IEEE Trans. Commun., vol. 63 , no. 9, pp. 3391-3404, Sept. 2015.

[12] Q. $\mathrm{Li}$ and $\mathrm{W}$. Ma, "Spatially selective artificial-noise aided transmit optimization for MISO multi-eves secrecy rate maximization,"IEEE Trans. Signal Process., vol. 61, no. 10, pp. 2704-2717, May 2013.

[13] S. Lohani, E. Hossain, and V. K. Bhargava, "On downlink resource allocation for SWIPT in small cells in a two-tier HetNet," IEEE Trans. Wireless Commun., vol. 15, no. 11, pp. 7709-7724, Nov. 2016.

[14] S. Akbar, Y. Deng, A. Nallanathan, M. Elkashlan, and A. H. Aghvami, "Simultaneous wireless information and power transfer in K-tier heterogeneous cellular networks," IEEE Trans. Wireless Commun., vol. 15 , no. 8, pp. 5804-5818, Aug. 2016.

[15] Y. Zhu, L. Wang, K.-K. Wong, S. Jin, and Z. Zheng, "Wireless power transfer in massive MIMO aided HetNets with user association," IEEE Trans. Commun., vol. 64, no. 10, pp. 4181-4195, Oct. 2016.

[16] M. Sheng, L. Wang, X. Wang, Y. Zhang, C. Xu, and J. Li, "Energy efficient beamforming in MISO heterogeneous cellular networks with wireless information and power transfer," IEEE J. Select. Areas Commun., vol. 34, no. 4, pp. 954-968, Apr. 2016.

[17] H. Zhang, S. Huang, C. Jiang, K. Long, V. C. M. Leung, and H. V. Poor "Energy efficient user association and power allocation in millimeterwave-based ultra dense networks with energy harvesting base stations," IEEE J. Select. Areas Commun., vol. 35, no. 9, pp. 1936-1947, Sept. 2017.

[18] J. Kim, H. Lee, C. Song, T. Oh, and I. Lee, "Sum throughput maximization for multi-user MIMO cognitive wireless powered communication networks," IEEE Trans. Wireless Commun., vol. 16, no. 2, pp. 913-923, Feb. 2017.

[19] T. Lv, H. Gao, and S. Yang, "Secrecy transmit beamforming for heterogeneous networks," IEEE J. Select. Areas Commun., vol. 33, no. 6, pp. 1154-1170, Jun. 2015.

[20] E. Tekin and A. Yener, "The Gaussian multiple access wire-tap channel," IEEE Trans. Inform. Theory, vol. 54, no. 12, pp. 5747-5755, Dec. 2008.

[21] Y. S. Shiu, S. Y. Chang, H. C. Wu, S. C. H. Huang, and H. H. Chen, "Physical layer security in wireless networks: A tutorial," IEEE Wireless Commun., vol. 18, no. 2, pp. 66-74, Apr. 2011.

[22] Y. Ren, T. Lv, H. G. 0001, and Y. Li, "Secure wireless information and power transfer in heterogeneous networks," IEEE Access, vol. 5, pp. 4967-4979, 2017.

[23] B. Li, Z. Fei, Z. Chu, and Y. Zhang, "Secure transmission for heterogeneous cellular networks with wireless information and power transfer," IEEE Systems Journal, vol. 12, no. 4, pp. 3755-3766, Dec. 2018.

[24] X. Hu, Li, K. Huang, Z. Fei, and K. K. Wong, "Secrecy energy efficiency in wireless powered heterogeneous networks: A distributed ADMM approach," IEEE Access, vol. 6, pp. 20609-20624, 2018.
[25] D. W. K. Ng, E. S. Lo, and R. Schober, "Multi-objective resource allocation for secure communication in cognitive radio networks with wireless information and power transfer," IEEE Trans. Veh. Techno., vol. 65, no. 5, pp. 3166-3184, May 2016.

[26] Y. Wu, X. Chen, C. Yuen, and C. Zhong, "Robust resource allocation for secrecy wireless powered communication networks," IEEE Commun. Letters, vol. 20, no. 12, pp. 2430-2433, Dec. 2016.

[27] F. Parzysz, M. Di Renzo and C. Verikoukis,"Power-availability-aware cell association for energy-harvesting small-cell base stations," IEEE Trans. Wireless Commun., vol. 16, no. 4, pp. 2409-2422, Apr. 2017.

[28] K. Wang, A. M. So, T. Chang, W. Ma and C. Chi,"Outage constrained robust transmit optimization for multiuser MISO downlinks: Tractable approximations by conic optimization," IEEE Trans. Signal Process., vol. 62, no. 21, pp. 5690-5705, Nov. 2014.

[29] Q. Li and W. K. Ma, "Optimal and robust transmit designs for MISO channel secrecy by semidefinite programming," IEEE Trans. Signal Process., vol. 59, no. 8, pp. 3799-3812, Aug. 2011.

[30] S. Boyd and L. Vandenberghe, Convex Optimization. Cambridge, U.K.: Cambridge Univ. Press, 2004.

[31] Z. Q. Luo, J. F. Sturm, and S. Z. Zhang, "Multivariate nonnegative quadratic mappings," SIAM J. Optim., vol. 14, no. 4, pp. 1140-1162, 2004.

[32] S. Ma, M. Hong, E. Song, X. Wang, and D. Sun, "Outage constrained robust secure transmission for MISO wiretap channels," IEEE Trans. Wireless Commun., vol. 13, no. 10, pp. 5558-5570, Oct. 2014.

[33] S. S. Christensen, R. Agarwal, E. de Carvalho, and J. M. Cioffi, "Weighted sum-rate maximization using weighted MMSE for MIMOBC beamforming design," IEEE Trans. Wireless Commun., vol. 7, no. 12, pp. 4792-4799, 2008.

[34] A. Ben-Tal and A. Nemirovski, "Lectures on modern convex optimization: analysis, algorithms, and engineering applications," ser. MPSSIAM series on optimization, Philadelphia, PA, USA: SIAM, 2001.

[35] A. Marshall and I. Olkin, Inequalities: theory of majorization and its applications. Academic Press New York, 1979.

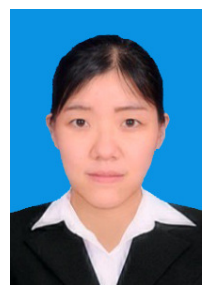

Shiqi Gong (S'14) received the B.S. degree in Electronic Engineering in 2014 from Beijing Institute of Technology, Beijing, China. Currently, she is pursuing the Ph.D. degree with the School of Electronic and Information, Beijing Institute of Technology. She also served as the Research Assistant of the University of Macau Faculty of Science and Technology. Her research interests are in the area of signal processing, physical-layer security, resource allocation, and convex optimization.

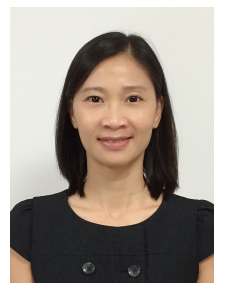

Shaodan Ma received her double Bachelor degrees in Science and Economics, and her Master degree of Engineering, from Nankai University, Tianjin, China,in 1999 and 2002 respectively. She obtained her $\mathrm{Ph} . \mathrm{D}$. degree in electrical and electronic engineering from the University of Hong Kong, Hong Kong, in 2006. From 2006 to 2011, she was a Postdoctoral Fellow in the University of Hong Kong. Since August 2011, she has been with the University of Macau and is now an Associate Professor there. She was a visiting scholar in Princeton University in 2010. Her research interests are in the general areas of signal processing and communications, particularly, transceiver design, resource allocation and performance analysis. 


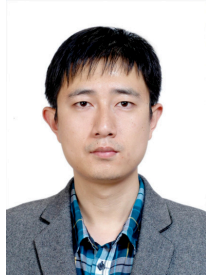

Chengwen Xing (Member, IEEE) received the B.Eng. degree from Xidian University, Xian, China, in 2005, and the Ph.D. degree from the University of Hong Kong, Hong Kong, China, in 2010. Since September 2010, he has been with the School of Information and Electronics, Beijing Institute of Technology, Beijing, China, where he is currently a Full Professor. From September 2012 to December 2012, he was a Visiting Scholar with the University of Macau, Macau, China. His current research interests include statistical signal processing, convex optimization, multivariate statistics, combinatorial optimization, massive MIMO systems, and high frequency band communication systems. He was an Associate Editor for the IEEE Transactions on Vehicular Technology 20132019, and currently serves as an Associate Editor for KSII Transactions on Internet and Information Systems, Transactions on Emerging Telecommunications Technologies, and China Communications.

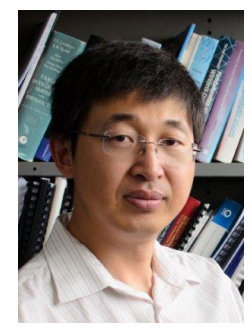

Yonghui Li (M'04-SM'09-F19) received his $\mathrm{PhD}$ degree in November 2002 from Beijing University of Aeronautics and Astronautics. Since 2003, he has been with the Centre of Excellence in Telecommunications, the University of Sydney, Australia. $\mathrm{He}$ is now a Professor and Director of Wireless Engineering Laboratory in School of Electrical and Information Engineering, University of Sydney. He is the recipient of the Australian Queen Elizabeth II Fellowship in 2008 and the Australian Future Fellowship in 2012. He is a Fellow of IEEE.

His current research interests are in the area of wireless communications, with a particular focus on MIMO, millimeter wave communications, machine to machine communications, coding techniques and cooperative communications. He holds a number of patents granted and pending in these fields. He is now an editor for IEEE Transactions on Communications, IEEE Transactions on Vehicular Technology. He also served as the guest editor for several IEEE journals, such as IEEE JSAC, IEEE Communications Magazine, IEEE IoT journal, IEEE Access. He received the best paper awards from IEEE International Conference on Communications (ICC) 2014, IEEE PIRMC 2017 and IEEE Wireless Days Conferences (WD) 2014.

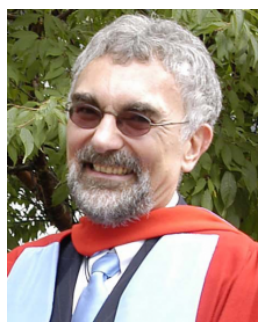

Lajos Hanzo (http://www-mobile.ecs.soton.ac.uk, https://en.wikipedia.org/wiki/Lajos_Hanzo) FREng, FIEEE, FIET, Fellow of EURASIP, DSc has received his Master degree and Doctorate in 1976 and 1983, respectively from the Technical University (TU) of Budapest. He was also awarded Honorary Doctorates by the TU of Budapest (2009) and by the University of Edinburgh (2015). He is a Foreign Member of the Hungarian Academy of Sciences and a former Editor-in-Chief of the IEEE Press. He has served as Governor of both IEEE ComSoc and of VTS. He has published 1900+ contributions at IEEE Xplore, 19 Wiley-IEEE Press books and has helped the fast-track career of $119 \mathrm{PhD}$ students. Over 40 of them are Professors at various stages of their careers in academia and many of them are leading scientists in the wireless industry. 